\title{
Polinização e dispersão de sementes em Myrtaceae do Brasil
}

\author{
ELIANA GRESSLER ${ }^{1}$, MARCO A. PIZO ${ }^{1,2}$ e L. PATRÍCIA C. MORELLATO ${ }^{1,3}$
}

(recebido: 6 de junho de 2005; aceito: 14 de setembro de 2006)

\begin{abstract}
Pollination and seed dispersal of Brazilian Myrtaceae). Myrtaceae is one of the most important plant families in Brazilian vegetation, especially forests. Its white, hermaphrodite flowers, with numerous stamens, and the fleshy fruits are exploited by a variety of animal species. This revision aimed to summarize the knowledge of the reproductive ecology of Brazilian Myrtaceae, bringing together information about the pollinators and seed dispersers of a great number of species. Data were compiled from the literature, complemented with unpublished information from the authors and other researchers. The majority of the pollination studies were carried out in Cerrado vegetation, whereas seed dispersal studies were conducted mainly on Atlantic Forest. Pollen is the major resource offered by Myrtaceae flowers. The flowers are visited mainly by bees, the pollinators of almost all species studied until now. The greatest number of visits to flowers is from Meliponinae and Bombinae bees (Apidae). Other insects such as flies and wasps also visit myrtaceous flowers, occasionally acting as pollinators. Bird pollination was reported for Acca sellowiana (O. Berg) Burret and Myrrhinium atropurpureum Schott, in which the main floral resource are the fleshy and sweet petals. Birds and monkeys are the main seed dispersers of Brazilian Myrtaceae, although other mammals, reptiles, fish and ants may interact and casually disperse seeds. Information on the pollination and seed dispersal agents of Myrtaceae in Brazil are still scarce, and its knowledge is essential to species preservation and the conservation of the Brazilian forests.
\end{abstract}

Key words - Myrtaceae, pollination, seed dispersal, reproductive ecology, tropical forest

RESUMO - (Polinização e dispersão de sementes em Myrtaceae do Brasil). Myrtaceae é uma das famílias de plantas mais importantes em várias formações vegetais brasileiras, especialmente nas florestas. Suas flores hermafroditas, de cor geralmente clara e com numerosos estames e os frutos carnosos são procurados por diversas espécies de animais. Esta revisão teve como objetivo sumarizar o conhecimento da ecologia reprodutiva das mirtáceas brasileiras, reunindo informações sobre os polinizadores e os dispersores de sementes do maior número de espécies. Os dados foram levantados da literatura, complementados com dados não publicados dos autores e outros pesquisadores. A maioria dos estudos de polinização foi desenvolvida no cerrado e os de dispersão na Floresta Atlântica. As flores de Myrtaceae são visitadas principalmente por abelhas, que coletam pólen e são os polinizadores da maioria das espécies. O maior número de visitas é de abelhas das subfamílias Meliponinae e Bombinae (Apidae). Outros insetos como moscas e vespas também visitam as flores das mirtáceas, poucas vezes atuando como polinizadores. A polinização por aves foi relatada em Acca sellowiana (O. Berg) Burret e Myrrhinium atropurpureum Schott, cujo recurso floral principal são as pétalas carnosas e doces. As aves e os macacos são os principais dispersores de sementes das mirtáceas brasileiras, sendo que outros mamíferos, répteis, peixes e formigas interagem de forma eventual, podendo contribuir para a dispersão de sementes. As informações sobre os agentes polinizadores e dispersores de sementes de Myrtaceae no Brasil ainda são escassas, sendo que seu conhecimento é essencial para a conservação das espécies e florestas brasileiras.

Palavras-chave - Myrtaceae, polinização, dispersão de sementes, ecologia reprodutiva, floresta tropical

\section{Introdução}

A família Myrtaceae compreende cerca de 100 gêneros e 3.500 espécies de árvores e arbustos que se

1. Universidade Estadual Paulista, Instituto de Biociências, Departamento de Botânica, Grupo de Fenologia e Dispersão de Sementes, Caixa Postal 199, 13506-900 Rio Claro, SP, Brasil.

2. Endereço atual: Universidade do Vale do Rio dos Sinos, Centro 2, 93022-000 São Leopoldo, RS, Brasil.

3. Autor para correspondência: pmorella@rc.unesp.br distribuem por todos os continentes, à exceção da Antártica, mas com nítida predominância nas regiões tropicais e subtropicais do mundo (Barroso 1991, Marchiori \& Sobral 1997). As mirtáceas têm sido organizadas tradicionalmente em duas subfamílias, Leptospermoideae e Myrtoideae, esta última incluindo todas as mirtáceas americanas, exceto o gênero monotípico Tepualia (Marchiori \& Sobral 1997). Atualmente, a nova classificação infra-família proposta por Wilson et al. (2005) reconhece duas subfamílias, Myrtoideae e Psiloxyloideae, e 17 tribos. Todas as 
mirtáceas brasileiras estão incluídas na Tribo Myrteae (sensu Wilson et al. 2005). Representada por aproximadamente 1.000 espécies, Myrtaceae é uma das famílias mais importantes do Brasil (Landrum \& Kawasaki 1997) destacando-se, com mais de uma centena de espécies, os gêneros Eugenia, Myrcia e Calyptranthes, enquanto o restante dos gêneros possui menos de 60 espécies brasileiras (Barroso \& Perón 1994, Landrum \& Kawasaki 1997).

Myrtaceae é uma das famílias lenhosas dominantes em várias formações vegetais brasileiras, especialmente na Floresta Atlântica onde mais de 50 espécies podem ocorrer sintopicamente (Peixoto \& Gentry 1990, Landrum \& Kawasaki 1997, Tabarelli \& Mantovani 1999, Oliveira Filho \& Fontes 2000, Guilherme et al. 2004). O entendimento da ecologia e história natural da Floresta Atlântica, aqui considerada em seu sentido mais amplo (Oliveira Filho \& Fontes 2000), um dos biomas mais ameaçados e de maior biodiversidade da Terra (Fonseca 1985, Morellato \& Haddad 2000), envolve necessariamente o conhecimento das mirtáceas.

As mirtáceas brasileiras geralmente não produzem madeiras valiosas, restringindo-se ao fornecimento de lenha, à utilização em pequenas peças ou objetos e outras formas de uso local (Marchiori \& Sobral 1997). Por outro lado, há numerosas espécies frutíferas, algumas exploradas comercialmente (e.g. a goiabeira, Psidium guajava L., a jabuticabeira, Myrciaria cauliflora (Mart.) O. Berg, e a pitangueira, Eugenia uniflora L.). Essas espécies representam apenas uma pequena fração do grande potencial econômico da família, tendo em vista o grande número de frutos comestíveis produzidos por espécies não comerciais (Landrum \& Kawasaki 1997). Algumas espécies estão desaparecendo da natureza antes mesmo que se tenha conhecimento básico de sua biologia (Landrum \& Kawasaki 1997), como pode ser visto pelas listas de espécies de Myrtaceae ameaçadas no Brasil divulgadas recentemente (Biodiversitas 2006).

Apesar de sua importância na estrutura das florestas e outras formações vegetais nativas, estudos de cunho ecológico abordando especificamente as mirtáceas brasileiras ainda são escassos (Souza 1997, Fidalgo 2002, Pizo 2003, Sugahara \& Takaki 2004, Gressler 2005). A polinização por abelhas, com o pólen como recurso principal, parece ser o sistema predominante (Nic Lughadha \& Proença 1996), embora estudos detalhados tenham sido feitos para poucas espécies (Proença 1992, Proença \& Gibbs 1994, Maués \& Couturier 2002, Fidalgo 2002, Torenzan-Silingardi \& Oliveira 2004). Vários autores reconhecem a escassez de informações sobre a biologia reprodutiva dos representantes da subfamília Myrtoideae em geral (van Wyk \& Lowrey 1988, Beardsell et al. 1993, Proença \& Gibbs 1994, Torenzan-Silingardi \& Del-Claro 1998). Até a publicação do trabalho de Proença \& Gibbs (1994), não havia estudos disponíveis sobre aspectos amplos da biologia da polinização para qualquer grupo de mirtáceas neotropicais. Todas as espécies de Myrtaceae brasileiras possuem frutos carnosos (Landrum \& Kawasaki 1997) cujas sementes são potencialmente dispersas por vertebrados frugívoros. As informações sobre quais vertebrados consumiriam frutos de mirtáceas ainda permanecem no campo das conjecturas, já que geralmente apenas observações eventuais estão disponíveis para poucas espécies (Kuhlmann \& Kuhn 1947, Pizo 2002). Ainda menor é o conhecimento sobre a eficiência de diferentes vertebrados como dispersores de sementes. Até o presente, o trabalho de Pizo (2002) é o único estudo abrangente que analisa os modos de dispersão e os dispersores de sementes de espécies de Myrtaceae da Floresta Pluvial Atlântica. O autor mostra que a grande variedade de características dos frutos (tamanho, cor, número e tamanho das sementes) apresentada pelas espécies de Myrtaceae está associada a um grupo diversificado de dispersores de sementes, principalmente aves e macacos.

A presente revisão tem por objetivo sumarizar o conhecimento da ecologia reprodutiva das mirtáceas brasileiras, reunindo informações especialmente sobre os polinizadores e os dispersores de sementes do maior número de espécies. Esperamos que esta revisão possa representar um ponto de partida para futuras pesquisas enfocando a ecologia da polinização e dispersão das espécies de Myrtaceae e de outras famílias importantes nas vegetações brasileiras.

Foram tomados como base para esta revisão os estudos com espécies nativas de Myrtaceae realizados no Brasil; fora do país foram considerados somente os estudos em que a espécie em questão ocorre naturalmente em território nacional. A maior parte dos dados foi levantada na literatura, complementada com observações de campo e dados não publicados dos autores e outros pesquisadores. No caso da polinização, devido à escassez de estudos, para alcançar uma visão mais abrangente na família foram incluídos comentários sobre algumas espécies de mirtáceas exóticas, quando pertinente. A polinização foi considerada efetiva quando mencionado que as estruturas reprodutivas da flor (estigma e estames) eram tocadas durante a visita do animal. Não foram incluídos registros sobre visitas da abelha africanizada Apis mellifera L., 1758 e sobre o sistema reprodutivo das mirtáceas. De uma forma geral, 
os dados de polinização levantados nesta revisão complementam aqueles apresentados por Nic Lughadha \& Proença (1996) para o Brasil.

Quanto aos dispersores das sementes de Myrtaceae no Brasil, também foram incluídas comunicações pessoais de outros pesquisadores. A maioria dos dados provém de estudos sobre a dieta de animais frugívoros e apenas os registros indicando a efetiva dispersão das sementes (i.e., engolir sementes inteiras sem quebrálas, regurgitar ou defecar sementes viáveis) foram considerados. Quando esses aspectos da frugivoria não eram relatados pelos autores, a possível ação do animal sobre as sementes foi inferida com base no comportamento de animais taxonomicamente relacionados e/ou na morfologia do aparelho digestivo (boca, dentição, presença de uma moela forte ou não). Não foram considerados registros de animais alimentando-se de frutos imaturos.

\section{A polinização em Myrtaceae}

Flores de Myrtaceae - As flores das mirtáceas brasileiras são hermafroditas, geralmente de cor branca, com estames numerosos, corola e cálice 4-5mero e ovário ínfero (Barroso 1991, Landrum \& Kawasaki 1997). A estrutura geral das flores varia pouco entre as espécies quando comparada com outras grandes famílias (Nic Lughadha \& Proença 1996; figuras 1-8). Flores menores são mais comuns, embora o tamanho varie de pequeno $(<1,5 \mathrm{~cm}$ de diâmetro), como em Calyptranthes e Myrcia (figuras 4, 7) a relativamente grande $(>2,0 \mathrm{~cm})$, como em $A c c a$ e Campomanesia (figuras 1, 5).

As pétalas e/ou os estames atuam como atrativos visuais aos polinizadores, mas os estames são, geralmente, as estruturas mais notáveis na flor aberta (Nic Lughadha \& Proença 1996), envolvidos na atração visual e olfativa dos polinizadores (figuras 1-8). O aroma também desempenha importante papel na atração dos polinizadores, sendo geralmente descrito como doce (Nic Lughadha \& Proença 1996), e produzido por osmóforos presentes principalmente no estigma, anteras, cálice e corola das flores (Souza 1997, Maués \& Couturier 2002). Em Myrcia cf. lingua (O. Berg) Mattos \& D. Legrand e Psidium cinereum Mart. ex DC., ocorrentes na área de cerrado no Estado de São Paulo, o forte odor adocicado é exalado com o início da liberação do pólen nas anteras (E. Gressler et al., dados não publicados). No gênero Myrcianthes, revisado por Grifo (1992), o aroma das flores foi descrito tanto como doce quanto levemente azedo.
O pólen é o principal recurso oferecido aos polinizadores, sendo o recurso primário pelo qual as abelhas, provavelmente o grupo mais importante de polinizadores de Myrtaceae, visitam as flores (Nic Lughadha \& Proença 1996). São poucas as evidências de produção de néctar, observada em Myrciaria dubia (Peters \& Vasquez 1986-1987, Maués \& Couturier 2002), Psidium guajava e Eugenia spp. (Ramalho et al. 1990), Myrciaria cauliflora (Malerbo et al. 1991) e Plinia glomerata (Pirani \& Cortopassi-Laurino 1993), consistindo no principal recurso oferecido aos visitantes em espécies do gênero Myrcianthes (Grifo 1992). A abertura das flores ocorre usualmente no início da manhã (entre $4 \mathrm{~h} 00$ e 6h00) e a flor dura, em geral, apenas um dia (Peters \& Vasquez 1986-1987, Proença \& Gibbs 1994, Fidalgo 2002, Maués \& Couturier 2002, E. Gressler, et al., dados não publicados).

Polinizadores e visitantes florais - Foram levantados os polinizadores e visitantes florais de 45 espécies de Myrtaceae brasileiras distribuídas em 14 gêneros (tabela 1). A maioria dos estudos sobre a polinização de mirtáceas brasileiras enfoca alguns poucos aspectos como biologia floral e a relação de visitantes observados, sendo raros aqueles que comprovam se os visitantes são realmente polinizadores efetivos da(s) espécie(s) sob investigação. Espécies ocorrentes no cerrado foram as mais estudadas, e abelhas foi o grupo mais comum de visitantes florais (tabela 1).

Abelhas - As abelhas foram os polinizadores mais freqüentes das mirtáceas ocorrentes no Brasil (35 espécies; tabela 1), sendo o maior número das visitas de abelhas da família Apidae, como verificado anteriormente por Nic Lughadha \& Proença (1996) em sua revisão sobre a biologia reprodutiva de Myrtoideae. O grande número de registros de Meliponinae se deve, em parte, ao fato de muitos trabalhos investigarem as plantas (fontes de pólen/néctar) procuradas por abelhas dessa subfamília de Apidae (Absy \& Kerr 1977, Absy et al. 1980, Absy et al. 1984, Knoll \& ImperatrizFonseca 1987, Cortopassi-Laurino \& Ramalho 1988, Guibu et al. 1988, Ramalho et al. 1989, Marquez-Souza 1996, Carvalho et al. 1999, 2001, Ramalho 2004). Segundo Proença \& Gibbs (1994) a importância de Colletidae na polinização das Myrtaceae neotropicais pode estar sendo subestimada, pois Proença (1992) observou Ptiloglossa sp. (Colletidae) polinizando Siphoneugena densiflora O. Berg no pré-amanhecer, período não considerado nos estudos baseados unicamente em observações diurnas.

O cerrado reúne o maior número de estudos específicos sobre a biologia reprodutiva de mirtáceas 
(tabela 1), e as abelhas formam o principal grupo de polinizadores das mirtáceas na região central do Brasil, área de ocorrência natural da maior parte do cerrado no país (Proença \& Gibbs 1994, Oliveira \& Gibbs 2000). Estudos considerando os modos de polinização na comunidade de plantas do cerrado confirmam esta tendência (Silberbauer-Gottsberger \& Gottsberger 1988, Oliveira \& Gibbs 2000, 2002). O estudo de SilberbauerGottsberger \& Gottsberger (1988) incluiu 18 espécies de Myrtaceae, mas como os autores não distinguiram os polinizadores por espécie observada, seus dados não puderam ser adicionados à tabela 1. Em outro estudo amplo sobre a biologia reprodutiva de espécies do cerrado, Oliveira \& Gibbs (2000) observaram que Blepharocalyx salicifolius (Kunth) O. Berg (figura 2) e Siphoneugena densiflora são polinizadas por abelhas de tamanho médio a grande (Bombus, Apidae: Bombinae), como verificado por Proença \& Gibbs (1994), e espécies pequenas de Melipona e Trigona (Apidae: Meliponinae). Oliveira \& Paula (2001) sugeriram que pequenos insetos, incluindo abelhas, podem ser os polinizadores de Calyptranthes widgreniana O. Berg., Eugenia florida DC. e Myrcia rostrata DC. (figura 7) em floresta de galeria associada ao cerrado no Brasil central.

O estudo de Proença \& Gibbs (1994) foi o primeiro que abordou a biologia reprodutiva de um grupo de mirtáceas simpátricas, ocorrentes em uma área de cerrado no Brasil central. Abelhas do gênero Bombus spp. (incluindo B. atratus e B. morio) foram os prováveis principais polinizadores de sete das oito espécies investigadas. Torezan-Silingardi \& Del-Claro (1998) apontam Eulaema nigrita Lepeletier, 1841 (Apidae: Bombinae) como polinizador efetivo e Bombus sp. como polinizadores eventuais de Campomanesia pubescens (DC.) O. Berg (figura 5) em uma área de cerrado no Estado de São Paulo. No estudo de Proença \& Gibbs (1994), apenas Bombus spp. são incluídas como polinizadores desta espécie. Em outra área de cerrado e matas de galeria em Minas Gerais, Torenzan-Silingardi \& Oliveira (2004) observaram que as flores de Myrcia rostrata e M. tomentosa (Aubl.) DC. foram visitadas por diversas espécies de abelhas, sendo polinizadas por Scaptotrigona e Trigona (Apidae: Meliponinae) e abelhas da família Halictidae (exceto M. tomentosa), resultados similares aos de Oliveira \& Gibbs (2000).

Na região Norte do Brasil, a maioria dos estudos com mirtáceas nativas não trata especificamente da polinização, abordando a fenologia e listando espécies vegetais procuradas por abelhas, sendo conduzidos predominantemente em áreas onde são cultivadas. Em áreas de cultivo no Amazonas, várias espécies de insetos, incluindo abelhas e moscas (sirfídeos), visitaram as flores do araçá-boi, Eugenia stipitata McVaugh (figura 6), sendo as abelhas os polinizadores principais, em especial Meliponinae e Bombinae, e os gêneros mais comuns Eulaema e Melipona (Falcão et al. 1988, 2000). $\mathrm{Na}$ mesma área de estudo de Falcão et al. (1988), o maior número de visitas de insetos às flores do araçápera, Psidium acutangulum DC. foi da ordem Hymenoptera, e Eulaema bombiformis (Packard, 1869), E. mocsaryi (Friese, 1899), Melipona pseudocentris (Cockerel, 1912), M. lateralis Erichson, 1848, Megalopta sp. e Ptilotrigona lurida (Smith, 1854) foram as espécies de abelhas mais encontradas (Falcão et al. 1992). A maioria das abelhas visitantes do araçá-pera foi também observada no araçá-boi (Falcão et al. 1988, 2000) e no camu-camu, Myrciaria dubia (Kunth) McVaugh (Falcão et al. 1989). Em floresta de terra firme na Amazônia Central, os principais visitantes e polinizadores de 11 espécies de Myrtaceae foram as abelhas Megalopta sp., Melipona spp., Trigona spp., Epicharis sp. e espécies de Anthophoridae (Souza 1997, tabela 1). No Pará, Maués \& Couturier (2002) observaram que as abelhas sem ferrão (Apidae: Meliponinae) são os visitantes mais importantes e os polinizadores efetivos do camu-camu, Myrciaria dubia (figura 8), sendo Nannotrigona punctata (Smith, 1854), Trigona branneri Cockerell, 1912 e T. pallens (Fabricius, 1798) as espécies mais freqüentes. O pólen constitui a maior recompensa aos visitantes do camu-camu, havendo também produção de néctar e procura deste recurso pelas abelhas (Maués \& Couturier 2002). Na Amazônia peruana, Peters \& Vasquez (1986-1987) relatam que os nectários do camucamu exsudam uma fragrância doce que atrai as abelhas e que as flores são polinizadas por Melipona erbunea fuscopilosa (Moure \& Kerr, 1950) e Trigona postica (Latreille, 1807). Apesar da possibilidade de polinização pelo vento, as abelhas são consideradas os polinizadores mais importantes do camu-camu (Peters \& Vasquez 1986-1987, Maués \& Couturier 2002).

Entre as escassas informações para a Floresta Atlântica, no Sudeste e Sul do Brasil, Ramalho (2004) relata intensa visitação às flores de Myrcia tomentosa por várias abelhas (Apidae: Meliponinae), principalmente espécies de Melipona e Plebeia. Em diferentes localidades do Estado de São Paulo, Pirani \& CortopassiLaurino (1993) observaram a coleta de pólen e néctar de flores de Plinia glomerata por Bombus sp., Melipona quadrifasciata Lepeletier, 1836 e Tetragonisca 
angustula (Latreille, 1811) (Apidae: Bombinae e Meliponinae). A mamangava Bombus morio (Swederus, 1787) foi a abelha visitante mais comum de seis mirtáceas em floresta de planície litorânea em São Paulo (tabela 1), seguida pela abelha sem-ferrão Melipona rufiventris Lepeletier, 1836, sendo ambas consideradas os polinizadores efetivos (Fidalgo 2002). No Sul do Estado de São Paulo, abelhas da subfamília Meliponinae polinizaram Calycorectes australis D. Legrand (E. Gressler et al., dados não publicados; figura 3).

Os visitantes e polinizadores de várias espécies de reconhecida importância econômica foram estudados em diversas partes do país (tabela 1). Em um pomar na Bahia, os polinizadores potenciais da goiabeira (Psidium guajava) foram Nannotrigona punctata, Trigona spinipes (Fabricius, 1793) e Melipona scutellaris Latreille, 1811 (Apidae: Meliponinae), e da pitangueira (Eugenia uniflora) Melipona scutellaris e Trigona spinipes (Castro 2002). No interior de São Paulo, foi encontrado pólen da goiabeira nas bolotas de Partamona helleri (Friese, 1900) e Plebeia droryana (Friese, 1900) - Apidae: Meliponinae (Carvalho et al. 1999). As abelhas Bombus morio e B. atratus Franklin, 1913 (Apidae: Bombinae) foram observadas coletando pólen da goiabeira em vegetação secundária no Sudeste do Brasil (Camillo \& Garófalo 1989). Em plantações no interior de São Paulo, os insetos mais freqüentes nas flores da jabuticabeira (Myrciaria cauliflora) foram as abelhas Tetragonisca angustula e Trigona spinipes - Apidae: Meliponinae (Malerbo et al. 1991). Segundo estes autores, as flores da jabuticabeira atraem as abelhas exclusivamente para a coleta de pólen, sendo seu néctar pobre em açúcares. A goiabeira-serrana, Acca sellowiana (O. Berg) Burret (figura 1), uma frutífera de grande potencial comercial (Mattos 1986) que ocorre naturalmente do Norte do Rio Grande do Sul ao Paraná (Lorenzi 1998), é a mirtácea brasileira mais estudada quanto ao sistema de polinização, objetivando aumentar sua produtividade. A polinização por aves, sugerida por diversos autores (tabela 1), é descrita detalhadamente adiante.

Os primeiros casos de polinização por vibração, ou "buzz pollination" (Proença 1992, Endress 1994) em mirtáceas brasileiras foram constatados em Siphoneugena densiflora por Ptiloglossa sp. (Colletidae), em Myrcia torta DC. - antes Myrcia dictiophylla (O. Berg) Mattos \& D. Legrand - por Augochloropsis sp. (Halictidae) e em Myrcia rhodeosepala Kiaersk. e Blepharocalyx salicifolius por Bombus spp. (Apidae: Bombinae) por Proença (1992) e Proença \& Gibbs (1994). A polinização por vibração em espécies de Myrtaceae brasileiras foi considerada surpreendente por Proença (1992), pois as espécies possuem anteras de deiscência longitudinal e não poricidas, usualmente encontradas em outras famílias de plantas com polinização vibrátil. Esse comportamento de vibração dos estames foi comum em mirtáceas da Amazônia central, efetuado por sete espécies de abelhas: Melipona cf. captiosa Moure, 1962, Melipona fulva Lepeletier, 1836, Megalopta sp., Paratetrapedia sp., Xylocopa sp. e espécies de Anthophoridae (Souza 1997). Torezan-Silingardi \& Del-Claro (1998) observaram polinização por vibração em Campomanesia pubescens por Eulaema nigrita em uma área de cerrado no Estado de São Paulo. Recentemente, polinização por abelhas pela vibração dos estames, especialmente por Bombus morio, foi descrita para seis espécies de Myrtaceae de Floresta Atlântica (Fidalgo 2002).

Outros insetos - Os relatos sobre a visita de outras espécies de insetos que não abelhas às flores das mirtáceas brasileiras são relativamente escassos, e referem-se, principalmente, a moscas (em especial os sirfídeos), vespas e besouros. Na maioria dos estudos estes insetos foram considerados apenas visitantes florais ou polinizadores ocasionais das mirtáceas (tabela 1). Entretanto, em floresta de terra firme na Amazônia Central, depois das abelhas, a mosca Ormidia sp. (Syrphidae) foi o polinizador potencial mais importante de 10 espécies de Myrtaceae, em especial Myrciaria floribunda (H. West ex Willd.) O. Berg (Souza 1997). Duas espécies de formigas, Crematogaster sp. e Ectatoma quadridens (Fabricius, 1973), visitaram as flores de Psidium acutangulum na Amazônia, aparentemente não realizando a polinização (Falcão et al. 1992).

Aves - Em Myrtaceae, a ornitofilia é comum em espécies australianas, mas rara nas americanas (Roitman et al. 1997). Os únicos casos conhecidos dentre as mirtoídeas americanas ocorrem em Acca e Myrrhinium, dois gêneros neotropicais com numerosos estames robustos e vermelhos, semelhantes aos de muitas mirtáceas polinizadas por aves da Australásia (Nic Lughadha \& Proença 1996). As flores destes gêneros não produzem odor nem néctar e apresentam como recurso floral para os polinizadores as pétalas carnosas (Mattos 1986, Stewart 1987, Ducroquet 1993, Ducroquet \& Hickel 1997, Roitman et al. 1997) que mudam de cor e tornam-se doces e suculentas com a deiscência das anteras (Landrum 1986). Segundo Roitman et al. (1997), a polinização de Acca e Myrrhinium é um caso especial, pois a maioria dos 
casos conhecidos de ornitofilia, nos neotrópicos, relata aves que procuram néctar, como os beija-flores, e raramente aves passeriformes (Sazima et al. 1993, Sazima \& Sazima 1999).

No Brasil, os principais polinizadores da goiabeiraserrana, Acca sellowiana, são aves que tocam as flores ao se alimentarem de suas pétalas (Popenoe 1912), havendo atualmente vários registros de aves se alimentando das pétalas da goiabeira-serrana (Stewart 1986, 1987, Ducroquet \& Hickel 1997, figura 1). Visitas por beija-flores são raras em Acca e, quando ocorrem, as aves não conseguem polinizar devido aos bicos longos (Ducroquet \& Hickel 1997). Em área cultivada em Santa Catarina, várias espécies de aves frugívoras foram observadas alimentando-se de flores da goiabeiraserrana, as mais freqüentes sendo o sanhaço-azulão, Thraupis sayaca (L., 1766) e o sanhaço-papa-laranja, T. bonariensis (Gmelin, 1789), da família Emberizidae (Ducroquet \& Hickel 1997). Todas as espécies de aves capturadas carregavam pólen na cabeça, indicando serem os principais polinizadores de A. sellowiana naquela região (Ducroquet \& Hickel 1997), embora os insetos, presentes em menor número nas flores, também contribuam para a polinização (Ducroquet 1993). Para os insetos, a distância entre o estigma e o plano das anteras dificultaria a polinização (Ducroquet \& Hickel 1997). Todavia, Hickel \& Ducroquet (2000) relatam a presença de pólen da goiabeira-serrana aderido às patas das abelhas Trigona spinipes, Tetragonisca angustula, Plebeia sp., Bombus atratus (todas Apidae) e Xylocopa augusti Lepeletier, 1841 e X. frontalis (Olivier, 1789) (Anthophoridae), sendo que as três últimas espécies tocam o estigma das flores, podendo atuar como polinizadores. Ducroquet \& Hickel (1997) sugerem que, no Brasil, a maioria das aves que come os frutos de A. sellowiana também come suas pétalas.

$\mathrm{Na}$ Argentina, Roitman et al. (1997) observaram que o sabiá-laranjeira, Turdus rufiventris Vieillot, 1818 (Muscicapidae) e Saltator aurantiirostris Vieillot, 1817 (Emberizidae: Cardinalinae) foram as aves visitantes mais comuns de Myrrhinium atropurpureum Schott, atuando como polinizadores juntamente com outras espécies de aves menos freqüentes nas flores. Segundo estes autores, em $M$. atropurpureum, que também ocorre no Sul do Brasil, a prevalência da ornitofilia é reforçada pela redução no número de estames de polistêmone para diplostêmone (e conseqüentemente na quantidade de pólen oferecida), pela presença de pétalas carnosas e adocicadas e pela virtual ausência de visitas por insetos nativos.

Mamíferos - Nenhum registro de mirtáceas brasileiras polinizadas ou visitadas por mamíferos foi encontrado. $\mathrm{Na}$ Austrália, uma espécie de morcego é descrita como polinizador de menor importância de Syzygium tierneyanum (Muell.) Hartley \& Perry, que floresce à noite (Hopper 1980). Roitman et al. (1997) não descartam a possibilidade de quiropterofilia em Myrrhinium atropurpureum na Argentina, mas não realizaram observações noturnas.

Vento - A importância da polinização pelo vento é ainda incerta nas mirtáceas americanas. Em todos os estudos que sugerem a possibilidade de polinização pelo vento em mirtáceas brasileiras, as abelhas são consideradas os polinizadores mais importantes (Peters \& Vasquez 1986-1987, Grifo 1992, Degenhart et al. 2001, Maués \& Couturier 2002). Maués \& Couturier (2002) relatam que o pólen de Myrciaria dubia é relativamente pequeno se comparado ao de outras mirtáceas (Barth \& Barbosa 1972), com superfície granulosa e seca, o que tornaria fácil seu transporte pelo vento.

\section{A dispersão de sementes em Myrtaceae}

Frutos de Myrtaceae - Todas as espécies de mirtáceas ocorrentes no Brasil produzem frutos carnosos, ou seja, frutos com sementes envolvidas por uma polpa carnosa tipicamente rica em água e carboidratos e pobre em proteínas e lipídeos (Landrum \& Kawasaki 1997, Pizo 2002). O tamanho dos frutos varia enormemente, desde 0,4 até $7,5 \mathrm{~cm}$ de largura e de 0,4 até $8,0 \mathrm{~cm}$ de comprimento (M.A. Pizo, dados não publicados). A cor dos frutos quando maduros é igualmente variável, incluindo preto (atro-purpúreo), vermelho, amarelo, laranja, cinza e verde, com predomínio da primeira

Figures 1-16. Brazilian species of Myrtaceae. Flowers: 1. Acca sellowiana (O. Berg) Burret (photo: J.P.H.J. Ducroquet). 2. Blepharocalyx salicifolius (Kunth) O. Berg (photo: C.E.B. Proença). 3. Calycorectes australis D. Legrand. 4. Calyptranthes lanceolata O. Berg. 5. Campomanesia pubescens (DC.). O. Berg. 6. Eugenia stipitata McVaugh (photo: S.A.N. Ferreira). 7. Myrcia rostrata DC. (photo: H.M. Torenzan-Silingardi). 8. Myrciaria dubia (Kunth) McVaugh (photo: M.M. Maués). Fruits: 9. Campomanesia xanthocarpa O. Berg. 10. Eugenia cuprea (O. Berg) Mattos. 11. Eugenia mosenii (Kausel) Sobral. 12. Eugenia multicostata D. Legrand. 13. Gomidesia flagellaris D. Legrand. 14. Marlierea obscura O. Berg. 15. Myrcengenia myrcioides (Cambess.) O. Berg. 16. Psidium guajava L. Bars $=1 \mathrm{~cm}$. 

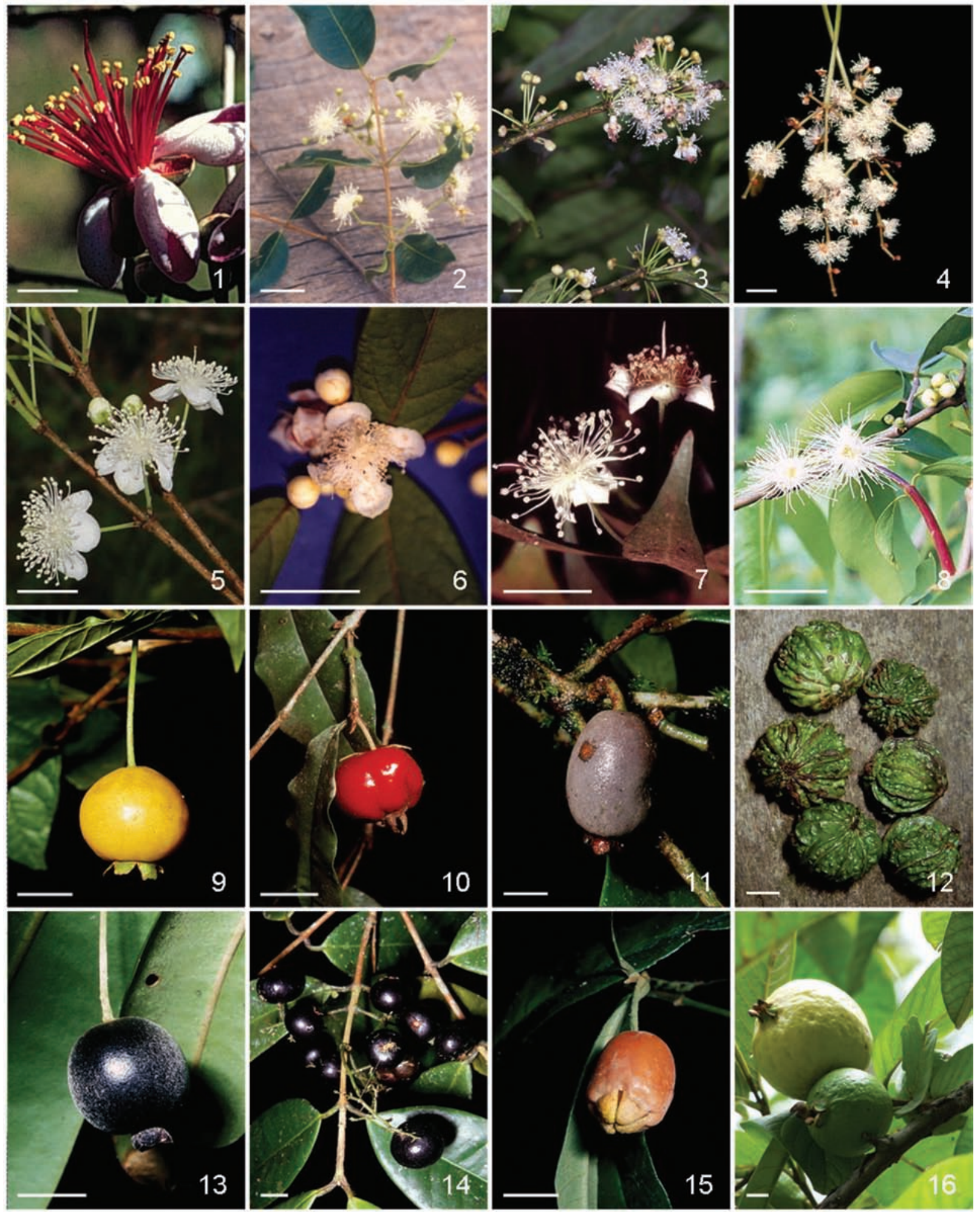

Figuras 1-16. Espécies de Myrtaceae brasileiras. Flores: 1. Acca sellowiana (O. Berg) Burret (foto: J.P.H.J. Ducroquet). 2. Blepharocalyx salicifolius (Kunth) O. Berg (foto: C.E.B. Proença). 3. Calycorectes australis D. Legrand. 4. Calypranthes lanceolata O. Berg. 5. Campomanesia pubescens (DC.). O. Berg. 6. Eugenia stipitata McVaugh (foto: S.A.N. Ferreira). 7. Myrcia rostrata DC. (foto: H.M. Torenzan-Silingardi). 8. Myrciaria dubia (Kunth) McVaugh (foto: M.M. Maués). Frutos: 9. Campomanesia xanthocarpa O. Berg. 10. Eugenia cuprea (O. Berg) Mattos. 11. Eugenia mosenii (Kausel) Sobral. 12. Eugenia multicostata D. Legrand. 13. Gomidesia flagellaris D. Legrand. 14. Marlierea obscura O. Berg. 15. Myrceugenia myrcioides (Cambess.) O. Berg. 16. Psidium guajava L. Barras $=1 \mathrm{~cm}$. 


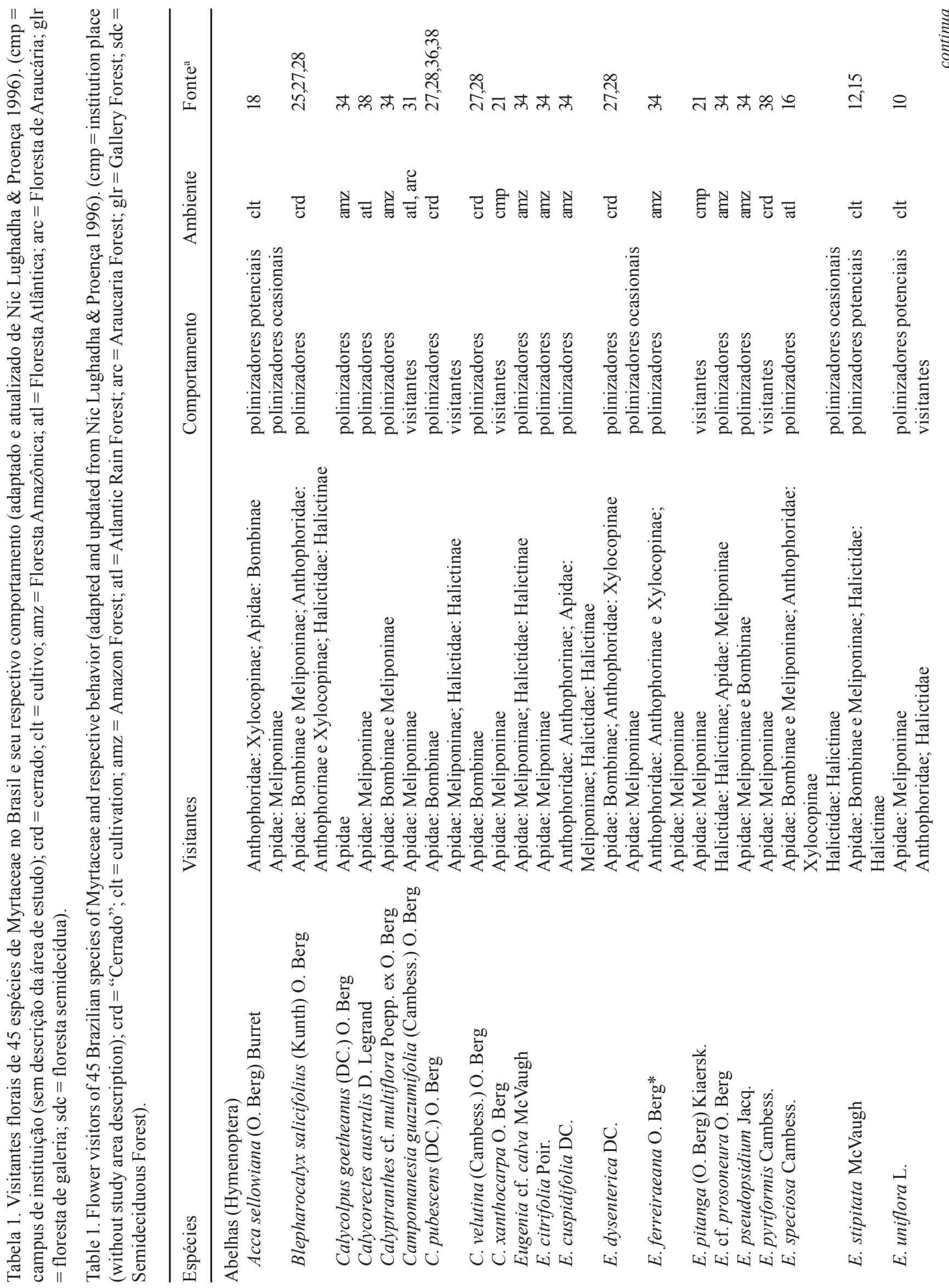




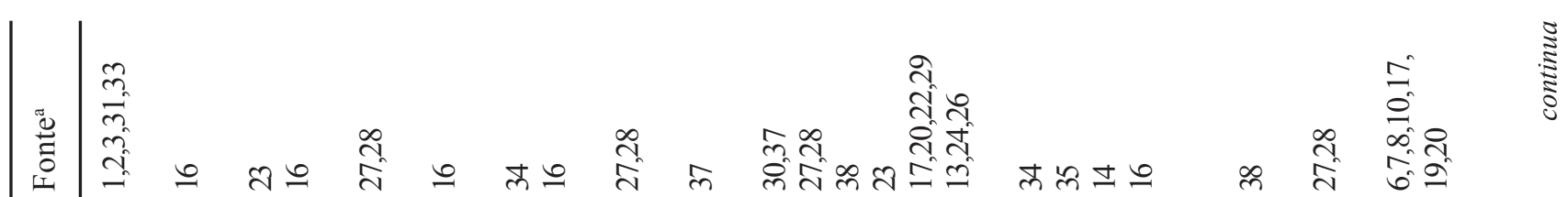

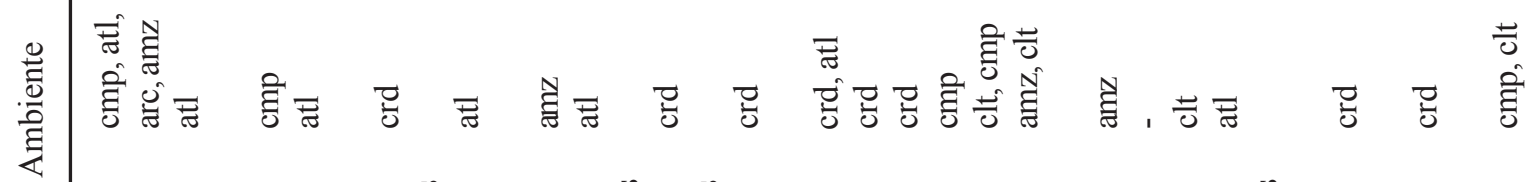<smiles>C[14CH]1CC1</smiles>

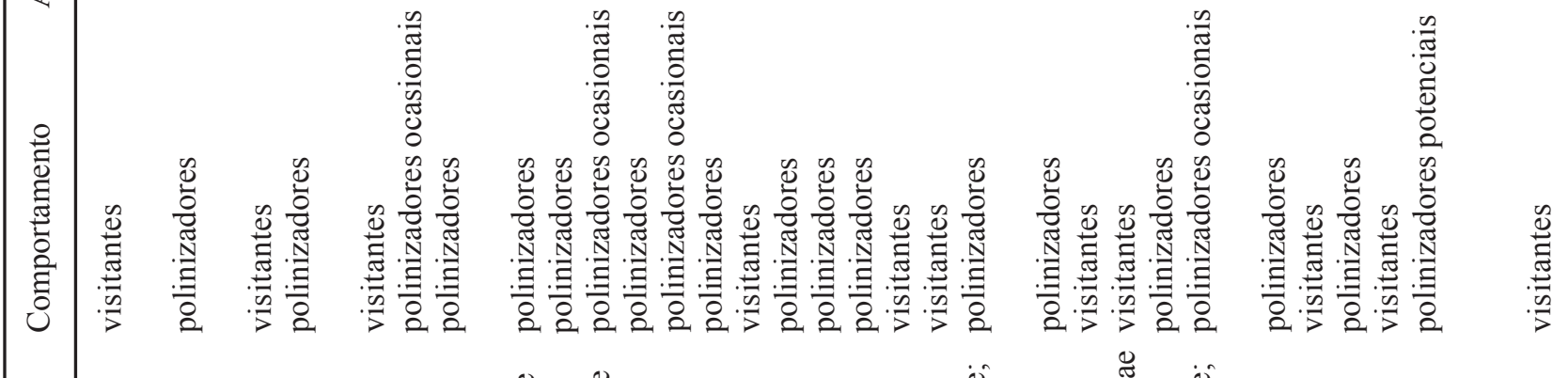
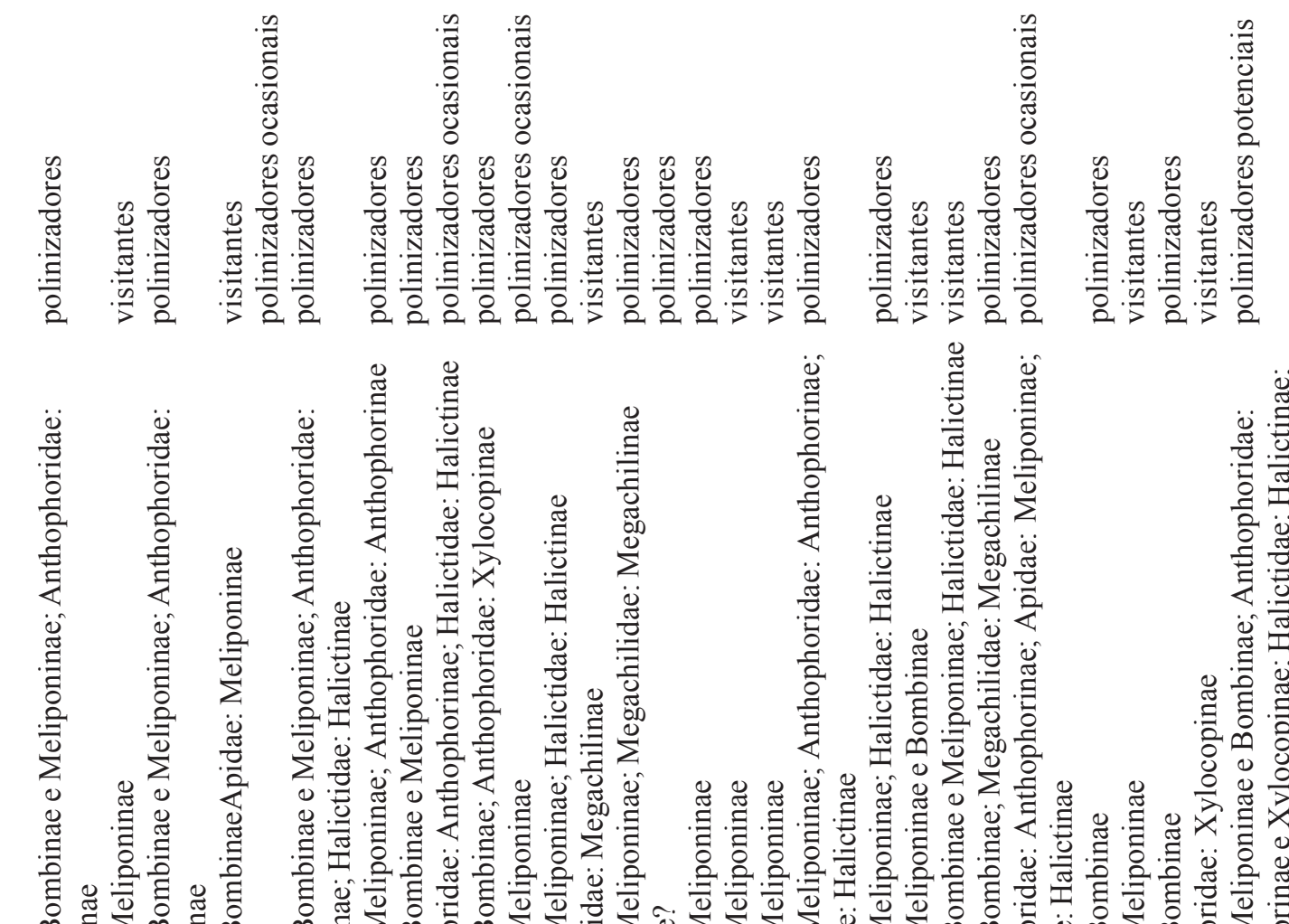

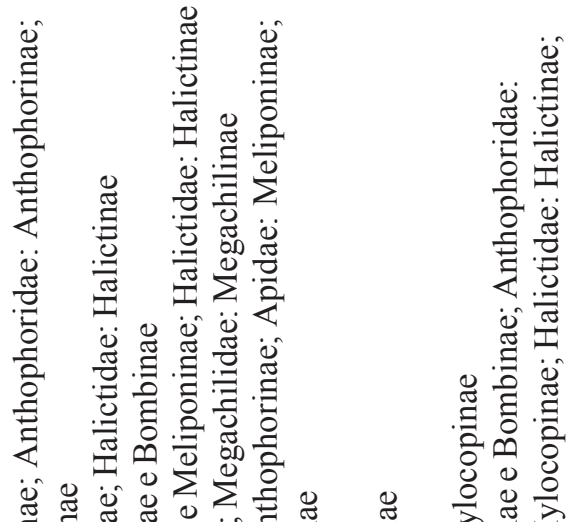

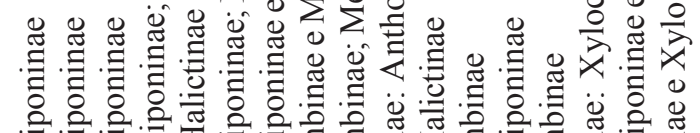

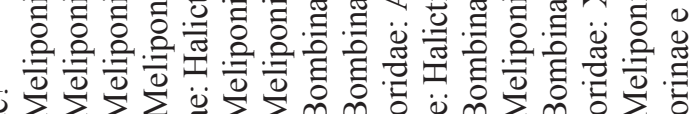

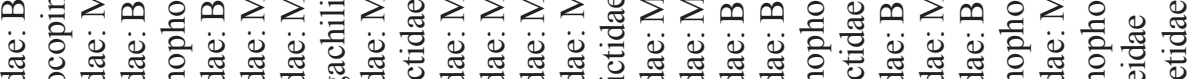

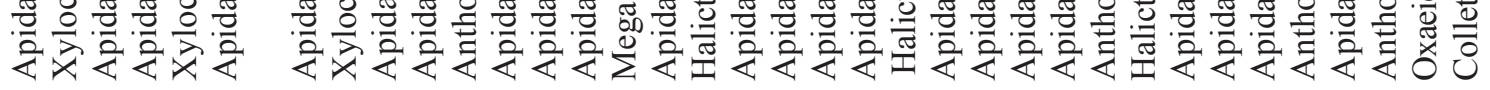

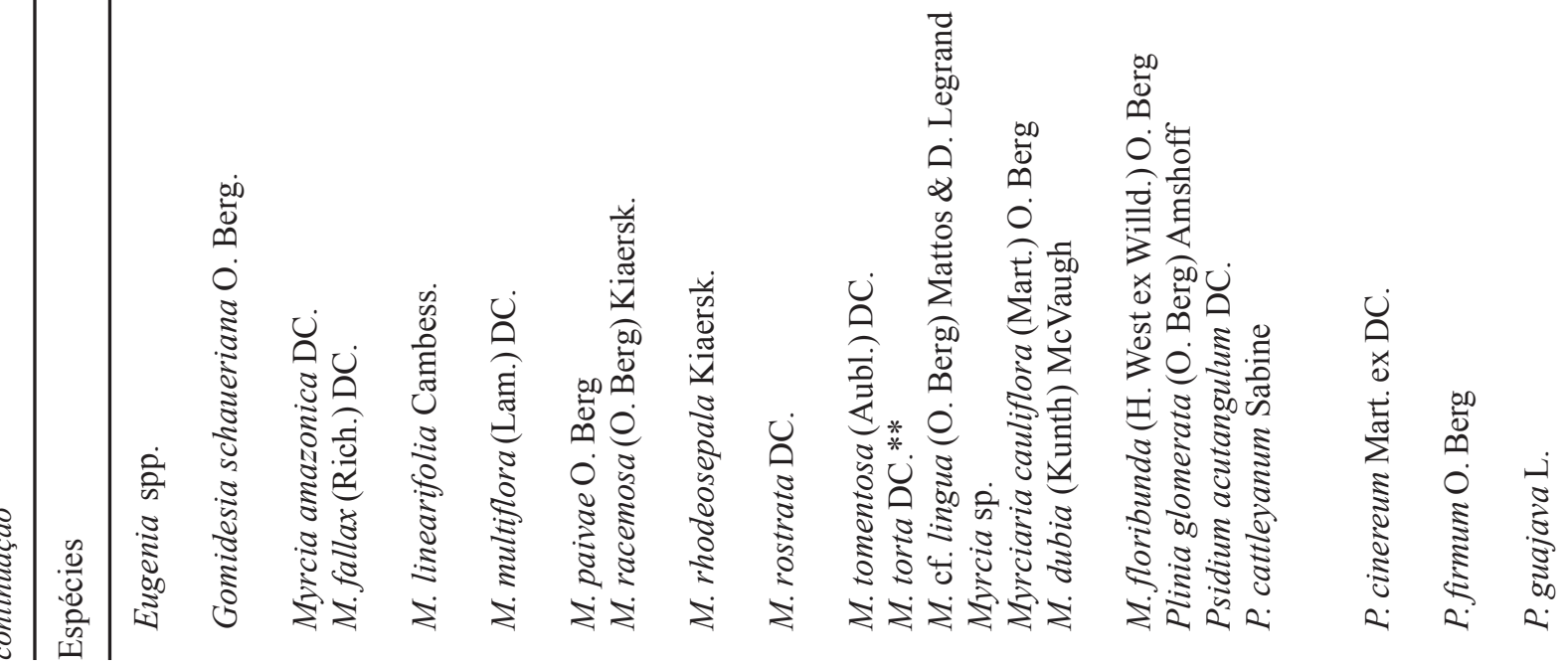




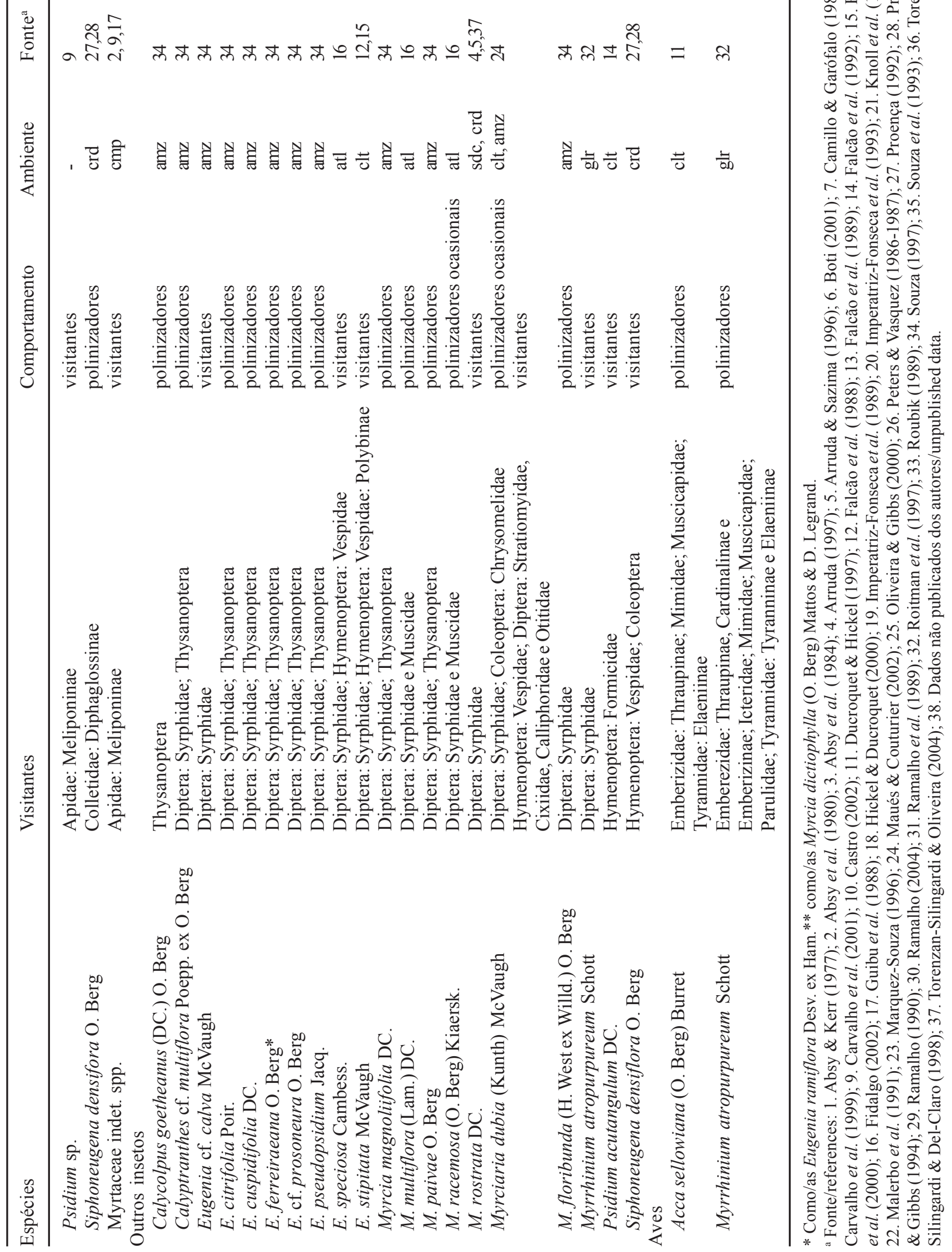


(figuras 9-16; Pizo 2002). Alguns gêneros são bastante homogêneos quanto à coloração dos frutos, como Campomanesia (figura 9) que sempre produz frutos amarelos, alaranjados ou verdes, enquanto outros como Eugenia são altamente variáveis (figuras 10-12). A maioria dos gêneros produz poucas sementes (1-3) por fruto, porém Campomanesia (4-18 sementes) e Psidium (até 250 sementes) fogem a este padrão (Pizo 2002). A testa que recobre as sementes é delgada na maioria dos gêneros, porém, bastante rígida em Campomanesia e, especialmente, em Psidium (Landrum \& Sharp 1989).

Dispersores de sementes - Os potenciais dispersores das sementes de 115 espécies de Myrtaceae nativas do Brasil, representando 17 gêneros, foram identificados (tabela 2). Os gêneros melhor representados foram Eugenia e Myrcia com 35 e 27 espécies, respectivamente. Alguns gêneros apresentaram ampla variedade de dispersores, com destaque para Psidium com registro para nove dos dez grupos de dispersores aqui considerados, enquanto outros são mais conservativos, como Campomanesia, Gomidesia e Myrcia (tabela 2). Ao contrário dos estudos de polinização, a maioria dos estudos com informações sobre os potenciais dispersores das sementes de mirtáceas no Brasil foram realizados na Floresta Atlântica, com alguns poucos estudos feitos em áreas de cerrado e da Amazônia.

Aves - As aves formam o principal grupo de dispersores das mirtáceas brasileiras (tabela 2). As aves registradas alimentando-se dos frutos de Myrtaceae variaram bastante de tamanho, desde $24 \mathrm{~g}$ - tangará-dançarino, Chiroxiphia caudata (Schaw \& Nodder, 1793) até aproximadamente $1,5 \mathrm{~kg}$ - jacutinga, Pipile jacutinga (Spix, 1825). O maior fruto disperso por aves é o de Eugenia mosenii (Kausel) Sobral (figura 11), com $2 \mathrm{~cm}$ de diâmetro, comido apenas por aves de grande porte (e.g. tucanos). Esse fruto está provavelmente próximo do limite máximo de tamanho para a dispersão por aves, pois a largura do bico das aves frugívoras neotropicais atuais atinge seu limite máximo em aproximadamente $3 \mathrm{~cm}$ (Wheelwright 1985, Silva \& Tabarelli 2000). Frutos maiores são comidos aos pedaços e, nestes casos, a dispersão efetiva das sementes depende de seu tamanho. Os grandes frutos do gênero Psidium possuem, tipicamente, várias (até 250) sementes pequenas (largura $<0,5 \mathrm{~cm}$ ) e são freqüentemente dispersos por aves (tabela 2) que, ao ingerirem porções da polpa, ingerem também algumas sementes.

Embora muitas das espécies de Myrtaceae dispersas por aves sejam também comidas por mamíferos, especialmente macacos, algumas são, provavelmente, dispersas exclusivamente pelas aves. Estas são plantas que habitam o sub-bosque das florestas e tipicamente produzem poucos $(<20)$ frutos maduros, como por exemplo Calyptranthes lanceolata O. Berg, Eugenia cuprea (O. Berg) Mattos (figura 10) e Gomidesia flagellaris D. Legrand (figura 13). Mamíferos - Os macacos são os mamíferos que mais freqüentemente dispersam as sementes de Myrtaceae (tabela 2). Eles variam enormemente de tamanho, desde aproximadamente $500 \mathrm{~g}$ - sagüis, Saguinus spp. - até cerca de $9 \mathrm{~kg}$ - mono-carvoeiro, Brachyteles arachnoides (É. Geoffroy, 1806). O gênero Campomanesia parece ser predominantemente disperso por mamíferos, especialmente macacos, em áreas florestais; oito das nove espécies para as quais há informação são dispersas por macacos. A única exceção foi $C$. pubescens, espécie de cerrado e campos, que é dispersa por mamíferos carnívoros como o lobo guará, Chrysocyon brachyurus (Illiger, 1815). As espécies de Campomanesia com registro de consumo por aves, C. guazumifolia (Cambess.) O. Berg e C. xanthocarpa O. Berg (figura 9), são dispersas por aves de grande porte, como o jacu, Penelope superciliaris Temminck, 1815. De fato, a maioria das espécies neste gênero possui frutos com diâmetro superior a 1,5 cm (Landrum 1986), portanto, superior à largura do bico da maioria das aves frugívoras (Wheelwright 1985). As aves, no entanto, podem comer alguns frutos de Campomanesia aos pedaços, evitando, porém, as sementes e, portanto, não realizando a dispersão (Landrum 1986).

Diferentemente dos Megachiroptera da região Paleotrópica (Marshall 1985), os morcegos neotropicais aparentemente alimentam-se de poucos frutos de Myrtaceae, pois apenas cinco espécies foram registradas (tabela 2). Para algumas espécies, no entanto, a ação dos morcegos parece aumentar o sucesso de germinação das sementes, como em Eugenia stictosepala Kiaersk. (M.A. Pizo, dados não publicados).

O coati, Nasua nasua (L., 1766), os cachorrosdo-mato, Cerdocyon thous (L., 1766) e Pseudalopex vetulus (Lund, 1842) e o lobo-guará foram os mamíferos carnívoros registrados como dispersores de sementes de Myrtaceae (tabela 2). Com exceção de plantas baixas encontradas em vegetações xerófitas como o cerrado, como Myrcia fallax (Rich.) DC. e Psidium guineense Sw., é provável que estes animais comam os frutos de maneira oportunista, à medida que os encontram caídos sob as plantas. O mesmo pode ser 
Tabela 2. Dispersores das sementes de 115 espécies de Myrtaceae no Brasil.

Table 2. Seed dispersers of 115 Brazilian species of Myrtaceae.

\begin{tabular}{|c|c|c|}
\hline Espécies & Dispersores & Fonte $^{\mathrm{a}}$ \\
\hline Blepharocalyx salicifolius (Kunth) O. Berg & aves & 73 \\
\hline Calycolpus goetheanus (DC.) O. Berg & aves & 60 \\
\hline Calycorectes australis D. Legrand & aves & 79 \\
\hline Calyptranthes concinna DC. & aves & 29,36 \\
\hline C. clusiifolia (Miq.) O. Berg & aves & 78 \\
\hline C. crebra McVaugh & macacos; peixes & 56,62 \\
\hline C. lanceolata O. Berg & aves & 79 \\
\hline C. lepida McVaugh & aves & 67 \\
\hline C. macrophylla O. Berg & macacos & 66 \\
\hline C. multiflora Poepp. ex O. Berg & macacos & 66 \\
\hline Campomanesia adamantium (Cambess.) O. Berg & macacos & 44,45 \\
\hline C. aromatica (Aubl.) Griseb. & macacos & 70 \\
\hline C. eugenioides (Cambess.) D. Legrand & macacos & 32 \\
\hline C. guaviroba (DC.) Kiaersk. & macacos & $7,44,45,54,55,64$ \\
\hline C. guazumifolia (Cambess.) D. Legrand & aves; macacos & 33,54 \\
\hline C. neriiflora (O. Berg) Nied. & macacos; ungulados & 28 \\
\hline C. pubescens (DC.) O. Berg & mamíferos carnívoros & 47 \\
\hline C. schlechtendahliana (O. Berg) Nied. & macacos & 65 \\
\hline C. xanthocarpa O. Berg & $\begin{array}{l}\text { aves; mamíferos carnívoros; macacos; } \\
\text { ungulados }\end{array}$ & $\begin{array}{l}5,13,20,23,24 \\
42,43\end{array}$ \\
\hline Eugenia acrensis McVaugh & macacos & 66 \\
\hline E. aurata O. Berg & aves; mamíferos carnívoros & 14,25 \\
\hline E. bimarginata $\mathrm{DC}$. & aves & 39 \\
\hline E. brasiliensis Lam. & aves; macacos & 1,23 \\
\hline E. brevipedunculata Kiaersk. & aves & 78 \\
\hline E. burkartiana (D. Legrand) D. Legrand & macacos & 55 \\
\hline E. cambucarana Kiaersk. & roedores & 57 \\
\hline E. cerasiflora Miq. & aves & 27 \\
\hline E. coffeifolia DC. & aves; macacos & 49,68 \\
\hline E. cuprea (O. Berg) Mattos & aves & 74 \\
\hline E. egensis DC. & macacos & 62 \\
\hline E. feijoi O. Berg & macacos & 69 \\
\hline E. flavescens DC. & macacos & 34 \\
\hline E. florida DC. & macacos & 42,54 \\
\hline E. inundata DC. & peixes & 56 \\
\hline E. involucrata DC. & macacos & 7,71 \\
\hline E. ligustrina (Sw.) Willd. & macacos & 75 \\
\hline E. melanogyna (D. Legrand) Sobral & morcegos & 79 \\
\hline E. mosenii (Kausel) Sobral & aves & 79 \\
\hline E. multicostata D. Legrand & roedores & 57 \\
\hline E. neoglomerata Sobral & aves & 79 \\
\hline E. neolanceolata Sobral & morcegos & 72 \\
\hline E. neoverrucosa Sobral & roedores & 57 \\
\hline E. oblongata Mattos \& D. Legrand & aves & 74 \\
\hline E. patrisii Vahl & aves; roedores & 35,59 \\
\hline E. pruinosa D. Legrand & macacos & 55 \\
\hline E. pseudopsidium Jacq. & aves & 59 \\
\hline E. punicifolia (Kunth) DC. & aves; macacos & 25,66 \\
\hline E. pyriformis Cambess. & macacos & $75,76,79$ \\
\hline
\end{tabular}


continuação

\begin{tabular}{|c|c|c|}
\hline Espécies & Dispersores & Fonte $^{\mathrm{a}}$ \\
\hline Eugenia ramboi D. Legrand & macacos & 24,42 \\
\hline E. rostrifolia D. Legrand & macacos & 13 \\
\hline E. stictosepala Kiaersk. & morcegos; macacos & 28,79 \\
\hline E. sulcata Spring ex Mart. & macacos & 53,54 \\
\hline E. umbelliflora O. Berg & aves & 8,26 \\
\hline E. uniflora $\mathrm{L}$. & $\begin{array}{l}\text { aves; mamíferos carnívoros; lagartos; } \\
\text { macacos }\end{array}$ & $\begin{array}{l}4,9,16,27,42 \\
47,63\end{array}$ \\
\hline Gomidesia affinis (Cambess.) D. Legrand & aves & 22,77 \\
\hline G. anacardiaefolia (Gardner) O. Berg & aves; macacos & 55,79 \\
\hline G. flagellaris D. Legrand & aves & 79 \\
\hline G. riedeliana $\mathrm{O}$. Berg & aves & 44,45 \\
\hline G. schaueriana O. Berg & aves & 8,77 \\
\hline G. sellowiana O. Berg & aves & 29,36 \\
\hline G. spectabilis (DC.) O. Berg & aves & 74,79 \\
\hline Hexachlamys itatiaiensis Mattos & aves & 76 \\
\hline $\begin{array}{l}\text { Marlierea eugeniopsoides (D. Legrand \& Kausel) } \\
\text { D. Legrand }\end{array}$ & macacos & 44,45 \\
\hline M. obscura O. Berg & aves & 74,79 \\
\hline M. regeliana $\mathrm{O}$. Berg & macacos & 79 \\
\hline M. reitzii D. Legrand & aves; macacos & 77 \\
\hline M. suaveolens Cambess. & aves; macacos & 44,45 \\
\hline M. tomentosa Cambess. & aves & 74,79 \\
\hline Myrceugenia myrcioides (Cambess.) O. Berg & aves; macacos & 55,74 \\
\hline Myrcia acuminatissima O. Berg & aves & 8 \\
\hline M. bicarinata (O. Berg) D. Legrand & formigas & 52 \\
\hline M. bombycina (O. Berg) Kiaersk. & aves & 29,37 \\
\hline M. bullata O. Berg & aves & 53 \\
\hline M. citrifolia (Aubl.) Urb. & aves & 67 \\
\hline M. dictyophylla (O. Berg) Mattos \& D. Legrand & aves & 2 \\
\hline M. fallax (Rich.) DC. & aves; mamíferos carnívoros; macacos & $7,12,30,36$ \\
\hline M. grandiflora (O. Berg) Nied. & macacos & 54,55 \\
\hline M. guianensis (Aubl.) DC. & aves & 67 \\
\hline M. heringii D. Legrand & macacos & 7 \\
\hline M. laruotteana Cambess. & aves & 36 \\
\hline M. lasiantha DC. & aves; mamíferos carnívoros & 14,25 \\
\hline M. leptoclada DC. & aves & 60,61 \\
\hline M. macrocarpa Barb. Rodr. & aves; macacos & 55 \\
\hline M. multiflora (Lam.) DC. & aves & 8 \\
\hline M. obtecta $(\mathrm{O} . \mathrm{Berg})$ & aves & 42 \\
\hline M. oxyoentophylla Kiaersk. & aves & 53 \\
\hline M. pubipetala Miq. & aves; mamíferos carnívoros; macacos & $7,55,64$ \\
\hline M. rostrata $\mathrm{DC}$. & aves; formigas; macacos & $32,33,36,52,64$ \\
\hline M. rufula Miq. & aves & 77 \\
\hline M. saxatilis (Amshoff) McVaugh & aves & 67 \\
\hline M. servata McVaugh & aves & 35 \\
\hline M. splendens (Sw.) DC. & aves; macacos & $48,58,66$ \\
\hline M. sylvatica (G. Mey.) DC. & aves & 67 \\
\hline M. tomentosa (Aubl.) DC. & aves & 25,27 \\
\hline M. torta DC. & aves & 25 \\
\hline M. variabilis DC. & macacos & 44,45 \\
\hline Myrcianthes pungens (O. Berg) D. Legrand & macacos & $6,10,71$ \\
\hline
\end{tabular}


continuação

\begin{tabular}{|c|c|c|}
\hline Espécies & Dispersores & Fonte $^{a}$ \\
\hline Myrciaria amazonica $\mathrm{O}$. Berg & macacos & 66 \\
\hline M. cauliflora (Mart.) O. Berg & mamíferos carnívoros; macacos & $33,46,47,76$ \\
\hline M. cuspidata $\mathrm{O}$. Berg & macacos & 20 \\
\hline M. floribunda (H. West ex Willd.) O. Berg & aves; macacos & $27,37,55$ \\
\hline M. tenella (DC.) O. Berg & aves & 37 \\
\hline Neomitranthes glomerata (D. Legrand) D. Legrand & aves & 79 \\
\hline N. obscura (DC.) N. Silveira & macacos & 38 \\
\hline Plinia complanata M. L. Kawasaki \& B. Holst & aves & 79 \\
\hline P. pauciflora M. L. Kawasaki \& B. Holst & aves & 79 \\
\hline Psidium acutangulum DC. & peixes & 31 \\
\hline P. cattleyanum Sabine & $\begin{array}{l}\text { aves; formigas; morcegos; macacos; } \\
\text { ungulados }\end{array}$ & $3,32,33,52$ \\
\hline P. cinereum Mart. ex DC. & mamíferos carnívoros & 47 \\
\hline P. fluviatile Rich. ex DC. & macacos & 48 \\
\hline P. guajava $\mathrm{L}$. & $\begin{array}{l}\text { aves; mamíferos carnívoros; morcegos; } \\
\text { lagartos; macacos; marsupiais; } \\
\text { ungulados }\end{array}$ & $\begin{array}{l}8,9,11,17,19,21 \\
42,47,60\end{array}$ \\
\hline P. guineense Sw. & mamíferos carnívoros & 18 \\
\hline P. laruotteanum Cambess. & mamíferos carnívoros & 15 \\
\hline P. longipetiolatum D. Legrand & macacos & 54,55 \\
\hline Siphoneugena densiflora O. Berg & aves; macacos & $44,45,79$ \\
\hline
\end{tabular}

${ }^{a}$ Fonte/references: 1. Alves \& Guix (1992); 2. Alves (1991); 3. Baker et al. (1998); 4. Bicca-Marques (1991); 5. Brisola (1993); 6. Brown et al. (1984); 7. Carvalho Júnior (1996); 8. Castro (1998); 9. Castro \& Galetti (2004); 10. Chitolina \& Sander (1981); 11. Cordero \& Nicolas (1987); 12. Costa (1998); 13. Cunha (1994); 14. Dalponte (1997); 15. Dalponte \& Lima (1999); 16. Dietz (1984); 17. Dinerstein (1986); 18. Facure \& Giaretta (1996); 19. Facure \& Monteiro-Filho (1996); 20. Fialho \& Setz (2000); 21. Fleming (1988); 22. Galetti \& Pizo (1996); 23. Galetti et al. (1997); 24. Gaspar (1997); 25. Gottsberger \& Silberbauer-Gottsberger (1983); 26. Guix \& Ruiz (1998); 27. Hasui \& Höfling (1998); 28. Izar (1999); 29. Kindel (1996); 30. Keeler-Wolf (1988); 31. Kubitzki \& Ziburski (1994); 32. Kuhlmann (1975); 33. Kuhlmann \& Kuhn (1947); 34. Lopes \& Ferrari (1994); 35. Macedo (1977); 36. Manhães (2003); 37. Marchiori \& Sobral (1997); 38. Martins (1997); 39. Martuscelli \& Olmos (1997); 40. Mendonça-Lima et al. (2001); 41. Mercolli \& Yanosky (1990); 42. Mikich (2000); 43. Miranda \& Passos (2004); 44. Moraes (1992a); 45. Moraes (1992b); 46. Motta-Júnior et al. (1994); 47. Motta-Júnior \& Martins (2002); 48. Nunes (1998); 49. Pack et al. (1996); 50. Passos (1992); 51. Passos (1997); 52. Passos \& Oliveira (2003); 53. Pereira et al. (1995); 54. Petroni (1993); 55. Petroni (2000); 56. Pires (1997); 57. Pizo (2002); 58. Riley \& Smith (1992); 59. Roth (1987); 60. Snow \& Snow (1971); 61. Snow (1962); 62. Soini (1987); 63. Souza et al. (1997); 64. Steinmetz (2000); 65. Strier (1991); 66. Terborgh (1983); 67. Théry \& Larpin (1993); 68. Théry et al. (1991); 69. van Roosmalem (1985a); 70. van Roosmalem (1985b); 71. Voss \& Sander (1980); 72. dados não publicados de Cinara S. B. Curra; 73. Iubatã P. de Faria; 74. Mauro Galetti; 75. Milene Martins; 76. Sandra B. Mikich; 77. Wesley R. Silva; 78. William Zaca; 79. Dados não publicados dos autores/unpublished data.

dito a respeito dos ungulados, especialmente a anta, Tapirus terrestris (L., 1758) e os veados, Mazama spp. Entretanto, sementes intactas foram encontradas nas fezes de todos estes animais, o que indica efetiva dispersão das sementes (tabela 2).

Poucas espécies têm suas sementes dispersas exclusivamente por roedores, como Eugenia cambucarana Kiaersk., E. multicostata D. Legrand (figura 12) e E. neoverrucosa Sobral (Pizo 2002). Os frutos destas espécies são morfologicamente homogêneos: são grandes ( $>3 \mathrm{~cm}$ de diâmetro), verdes ou amarelos, costados ou muricados, com uma a três sementes grandes e bastante rígidas. É provável que estas espécies sejam dispersas por cotias, Dasyprocta spp., e ratos-de-espinho, Proechimys spp. Na Amazônia foi registrada a dispersão de sementes, possivelmente secundária, de Eugenia patrisii Vahl por pacas, Agouti paca (L., 1766) e cutiarana, Myoprocta acouchy (Erxleben, 1777) (Macedo 1977). Obviamente não se pode descartar a possibilidade dos roedores eventualmente atuarem como dispersores secundários de Myrtaceae, assim como acontece com outras famílias de plantas (Forget \& Milleron 1991). O gambá, Didelphis marsupialis L., 1758 foi o único marsupial registrado alimentando-se de frutos de Myrtaceae (Cordero \& Nicolas 1987). 
Répteis - Sementes de Eugenia uniflora foram encontradas no estômago de Tupinambis merianae (Duméril \& Bibron, 1839), família Teiidae (Mercolli \& Yanosky 1990). Sementes desta espécie recolhidas das fezes de indivíduos cativos de T. merianae tiveram um sucesso de germinação superior a $75 \%$ (Castro \& Galetti 2004). A ingestão de frutos de Myrtaceae por répteis no Brasil, no entanto, provavelmente representa eventos esporádicos que ocorrem quando os animais encontram frutos caídos sob as plantas.

Peixes - Apenas três espécies foram registradas como potencialmente dispersas por peixes, todas em matas de várzea da região amazônica (tabela 2). Mais uma vez é provável que os peixes atuem apenas esporadicamente na dispersão das sementes de indivíduos de Myrtaceae cujos frutos caem naturalmente ou são derrubados na água por outros dispersores, como aves e mamíferos. Entretanto, será difícil avaliar a real importância dos peixes como dispersores de Myrtaceae encontradas em matas de várzea e de galeria até que maior número de estudos com este grupo de vertebrados, em geral muito mal conhecido quanto à dispersão de sementes, esteja disponível no Brasil.

Formigas - Embora não haja frutos de Myrtaceae primariamente adaptados à dispersão por formigas (mirmecocóricos, sensu van der Pijl 1982) no Brasil, estes insetos interagem com uma variedade de frutos que encontram no solo e podem, eventualmente, atuar como dispersores secundários de alguns deles, especialmente os menores ( $<1 \mathrm{~g}$; Pizo \& Oliveira 2000). Na restinga do Parque Estadual da Ilha do Cardoso, Estado de São Paulo, por exemplo, plântulas de Myrcia rostrata e Psidium cattleyanum Sabine estão significativamente associados aos ninhos de Odontomachus chelifer (Latreille, 1802), subfamília Ponerinae, formiga de grande porte $(\approx 1 \mathrm{~cm} \mathrm{de}$ comprimento) que carrega frutos caídos das árvores para seus ninhos a fim de alimentar as larvas e, posteriormente, descartam para fora do ninho sementes intactas e isentas de polpa (Passos \& Oliveira 2003).

\section{Conclusões e perspectivas}

A predominância de polinização por abelhas e dispersão de sementes por vertebrados frugívoros nas mirtáceas brasileiras, previsível com base em sua morfologia floral bastante conservativa e frutos exclusivamente carnosos, foi reafirmada nesta revisão. Diversificação maior foi observada em relação à dispersão de sementes. A variada morfologia dos frutos, principalmente quanto ao tamanho, coloração e número de sementes, permitiu identificar diferentes grupos de dispersores, de pequenas a grandes aves frugívoras, macacos e morcegos.

Myrtaceae é uma das famílias apícolas mais importantes do Brasil, sendo citada em quase todos os estudos que envolvem a determinação das espécies vegetais de interesse para abelhas. $O$ principal recurso floral oferecido aos visitantes é o pólen, eventualmente associado à presença de néctar. As flores geralmente pequenas das mirtáceas brasileiras, e das Myrtoideae americanas como um todo, são polinizadas principalmente por pequenos insetos, especialmente as abelhas, embora a polinização pelo vento (poucas evidências) e por aves, possa ocorrer de forma limitada. O tamanho da flor está diretamente relacionado ao tamanho da abelha visitante (Proença \& Gibbs 1994, E. Gressler et al., dados não publicados). A maioria das flores das mirtáceas brasileiras apresenta características da síndrome da melitofilia, confirmando a polinização por abelhas como a síndrome mais comum (Nic Lughadha \& Proença 1996). A polinização por vibração aparece como potencialmente importante em Myrtoideae e pode ser mais difundida do que se pensa. Trabalhos futuros podem confirmar a real importância da anemofilia na família.

Ainda há poucos estudos sobre a polinização de mirtáceas sul-americanas, visto que muitas espécies ainda são desconhecidas, notadamente na Floresta Atlântica brasileira, grande centro de diversificação desta família (Landrum \& Kawasaki 1997). Após dez anos da realização da revisão sobre a biologia reprodutiva de Myrtoideae por Nic Lughadha \& Proença (1996), ainda permanecem em aberto as questões levantadas por esses autores relativas à freqüência de ocorrência da polinização por vibração e como evoluiu a polinização por aves em que as pétalas são oferecidas como recompensa.

Devido à rápida expansão geográfica da abelha africanizada Apis mellifera na região neotropical, uma preocupação básica dos biólogos passou a ser o seu impacto sobre a fauna de abelhas nativas, incluindo as centenas de espécies da subfamília Meliponinae (Apidae) que são os principais polinizadores das mirtáceas brasileiras (Imperatriz-Fonseca et al. 1993, Nic Lughadha \& Proença 1996, tabela 1). Apis mellifera tem a capacidade de dominar os recursos florais e deslocar os polinizadores nativos e muitas vezes é considerada "ladra de pólen", pois apesar de realizar visitas freqüentes à flores, não as poliniza e esgota seus recursos (Roubik 1978, 1979, Schaeffer et al. 1983, Roubik et al. 1986, Torenzan-Silingardi \& Del-Claro 
1998). Além disso, Malerbo et al. (1991) observaram que a freqüência de visitas das abelhas nativas aumenta conforme diminui a freqüência de $A$. mellifera nas flores da jabuticabeira (Myrciaria cauliflora), confirmando dados anteriores de que a presença da abelha exótica desestimula a coleta pelas abelhas nativas brasileiras. Arruda (1997) relata que $A$. mellifera, juntamente com as vespas, são responsáveis pelo elevado deslocamento de sirfídeos (moscas) das flores. Dessa forma, ao reduzir a variedade de visitantes e a disponibilidade de alimento para os polinizadores nativos, $A$. mellifera pode afetar a polinização de espécies de mirtáceas nativas, uma possibilidade que deve ser investigada seriamente.

O levantamento dos agentes dispersores de uma família tão diversa quanto Myrtaceae e que ocorre em uma área geográfica extensa como o Brasil ainda é, naturalmente, incompleto. As informações da literatura são escassas e freqüentemente provêm de estudos focados nos animais e não nas plantas. Além disso, alguns grupos de animais, como aves e macacos, são mais freqüentemente estudados que outros (e.g. outros mamíferos, peixes), o que adiciona certo viés ao conjunto de dados. Apesar disso, alguns padrões surgiram e merecem destaque.

Aves e macacos são os principais dispersores de Myrtaceae no Brasil e, a julgar por dados não publicados que pudemos levantar de outros países, são também os principais dispersores em toda a região neotropical. Um estudo anterior mostrou que algumas características morfológicas distinguem os frutos de Myrtaceae dispersos por estes dois grupos de animais (Pizo 2002). De especial importância são os tamanhos do fruto e de suas sementes. Frutos pequenos (i.e., $<1 \mathrm{~cm}$ diâmetro) são freqüentemente dispersos por aves, que somente podem atuar como dispersoras de frutos grandes (i.e., diâmetro $>3 \mathrm{~cm}$ ) se estes tiverem sementes pequenas (e.g. Psidium). Os macacos, por sua vez, são menos limitados por estas características morfológicas e potencialmente podem dispersar grande variedade de frutos.

Os outros dispersores registrados neste levantamento aparentemente interagem apenas de forma eventual e pontual com os frutos de Myrtaceae. Deve-se ressaltar, entretanto, que o presente levantamento está fortemente baseado em estudos feitos em áreas florestais, especialmente da Floresta Atlântica. É possível que em áreas com vegetação mais baixa (e.g. o cerrado em suas várias fisionomias) os mamíferos não-primatas, especialmente os canídeos, ganhem importância como dispersores de Myrtaceae (Gottsberger \& Silberbauer-Gottsberger 1983).
Conhecer os agentes dispersores das plantas é essencial para qualquer programa que vise a preservação in situ de suas populações. Apesar disso, espécies de Myrtaceae com potencial econômico estão desaparecendo da natureza sem que saibamos quais são seus agentes dispersores (e.g. Campomanesia phaea; Kawasaki \& Landrum 1997). Outras podem estar ameaçadas devido ao desaparecimento dos dispersores de suas sementes (Silva \& Tabarelli 2000, Cordeiro \& Howe 2003). Por exemplo, Eugenia mosenii e E. melanogyna (D. Legrand) Sobral parecem depender fortemente de aves frugívoras de grande porte para dispersão de suas sementes, enquanto Eugenia cambucarana, E. multicostata e E. neoverrucosa são dispersas exclusivamente por roedores de médio a grande porte, especialmente cutias (Pizo 2002). Estes são exatamente os animais que, devido à caça e/ou alteração do hábitat (Willis 1979, Redford 1992), desaparecem de ambientes perturbados (Asquith et al. 1999). Assim, podemos prever o desaparecimento a longo prazo ou, no mínimo, a alteração dos padrões de recrutamento nas populações destas plantas que ocorrem nos inúmeros fragmentos de vegetação nativa espalhados pelo Brasil (Ranta et al. 1998).

Agradecimentos - Somos gratos a Carolyn E. B. Proença e outro revisor anônimo pela análise do manuscrito e sugestões e à Fundação Florestal do Estado de São Paulo por permitir a realização de trabalhos de campo no Parque Estadual Intervales, onde parte dos dados aqui apresentados foi coletada. Lúcia Kawasaki e Valesca B. Zipparro foram fundamentais na solução de problemas taxonômicos. Adriana A. Fidalgo, Cinara S.B. Curra, Flávio H.G. Rodrigues, Iubatã P. de Faria, Maria Anália D. Souza, Marilise Krügel, Mauro Galetti, Milene Martins, Sandra B. Mikich, Wesley R. Silva e William Zaca gentilmente forneceram dados não publicados. Carolyn E.B. Proença, Jean P.H. Ducroquet, Sidney A.N. Ferreira, Helena M. Torenzan-Silingardi e Márcia M. Maués gentilmente cederam fotos de Myrtaceae. Este estudo foi financiado pela Fapesp, International Foundation for Science, British Ecological Society, Idea Wild e CNPq. E. Gressler recebeu bolsa de mestrado e é bolsista de doutorado da Fapesp; M.A. Pizo recebeu bolsa de pós-doutorado da Fapesp e L.P.C. Morellato e M.A. Pizo são bolsistas de Produtividade em Pesquisa do CNPq.

\section{Referências bibliográficas}

ABSY, M.L. \& KERR, W.E. 1977. Algumas plantas visitadas para obtenção de pólen por operárias de Melipona seminigra merrillae em Manaus. Acta Amazonica 7:309-315.

ABSY, M.L., BEZERRA, E.B. \& KERR, W.E. 1980. Plantas nectaríferas utilizadas por duas espécies de Melipona na Amazônia. Acta Amazonica 10:271-281. 
ABSY, M.L., CAMARGO, J.M.F., KERR, W.E. \& MIRANDA, I.P.A. 1984. Espécies de plantas visitadas por Meliponinae (Hymenoptera; Apoidea) para coleta de pólen na região do médio Amazonas. Revista Brasileira de Biologia 44:227-237.

ALVES, I.M.S.C. \& GUIX, J.C. 1992. Feeding habits of Alouatta caraya in a semi-natural area (SE Brazil). Mammalia 56:469-472.

ALVES, M.A.S. 1991. Dieta e táticas de forrageamento de Neothraupis fasciata em cerrado no Distrito Federal, Brasil (Passeriformes, Emberezidae). Ararajuba 2:25-29.

ARRUDA, V.L.V. 1997. Uso de recursos florais por sirfídeos (Diptera, Syrphidae) e interações com outros visitantes. Naturalia 22:163-178.

ARRUDA, V.L.V. \& SAZIMA, M. 1996. Flores visitadas por sirfídeos (Diptera, Syrphidae) em mata mesófila de Campinas, SP. Revista Brasileira de Botânica 19:109-117.

ASQUITH, N.M., TERBORGH, J.,ARNOLD,A.E. \& RIVEROS, M. 1999. The fruits the agouti ate: Hymenaea courbaril seed fate when its disperser is absent. Journal of Tropical Ecology 15:229-235.

BAKER, H.G., BAKER, I. \& HODGES, S.A. 1998. Sugar composition of nectars and fruits consumed by birds and bats in the tropics and subtropics. Biotropica 30:559-586.

BARROSO, G.M. 1991. Sistemática de Angiospermas do Brasil. Universidade Federal de Viçosa, Viçosa, v. 2.

BARROSO, G.M. \& PERÓN, V. 1994. Myrtaceae. In Reserva Ecológica de Macaé de Cima, Nova Friburgo, RJ. Aspectos florísticos das espécies vasculares. (M.P.M. Lima \& R.R. Guedes-Bruni, eds.). Jardim Botânico do Rio de Janeiro, Rio de Janeiro, v.1, p.261-302.

BARTH, O.M. \& BARBOSA, A.F. 1972. Catálogo sistemático dos pólen das plantas arbóreas do Brasil Meridional. XV. Myrtaceae. Memórias do Instituto Oswaldo Cruz 70:467-495.

BEARDSELL, D.V., O’BRIEN, S.P., WILLIAMS, E.G., KNOX, R.B. \& CALDER, D.M. 1993. Reproductive biology of Australian Myrtaceae. Australian Journal of Botany 41:511-526.

BICCA-MARQUES, J.C. 1991. Ecologia e comportamento de um grupo de bugios-pretos Alouatta caraya (Primates, Cebidae) em Alegrete, RS, Brasil. Dissertação de mestrado, Universidade de Brasília, Brasília.

BIODIVERSITAS. 2006. Lista da flora ameaçada de extinção com ocorrência no Brasil IUCN. http:// www.biodiversitas.org.br/floraBr/listas_flora.asp (acesso em 15/02/2006).

BOTI, J.B. 2001. Polinização entomófila da goiabeira (Psidium guajava L., Myrtaceae): influência da distância de fragmentos florestais em Santa Teresa, Espírito Santo. Dissertação de mestrado, Universidade Federal de Viçosa, Viçosa.
BRISOLA, L.M.L. 1993. Estudo do hábito alimentar de Tapirus terrestris (ordem Perissodactyla) e sua atuação como dispersor de sementes no Parque Estadual do Morro do Diabo. Monografia de bacharelado, Universidade Estadual Paulista, Rio Claro.

BROWN, A., CHALUKIAN, S. \& MALMIERCA, L. 1984. Habitat y alimentacion de Cebus apella en el N.O. Argentino y la disponibilidad de frutos en el doseo arboreo. Revista del Museo Argentino de Ciência Naturales "Bernardino Rivadavia” 13:273-284.

CAMILLO, E. \& GARÓFALO, C.A. 1989. Analysis of the niche of two sympatric species of Bombus (Hymenoptera, Apidae) in south-eastern Brazil. Journal of Tropical Ecology 5:81-92.

CARVALHO, C.A.L., MARCHINI, L.C. \& ROS, P.B. 1999. Fontes de pólen utilizadas por Apis mellifera L. e algumas espécies de Trigonini (Apidae) em Piracicaba (SP). Bragantia 58:49-56.

CARVALHO, C.A.L., MORETI, A.C.C.C., MARCHINI, L.C., ALVES, R.M.O. \& OLIVEIRA, P.C.F. 2001. Pollen spectrum of honey of "uruçu" bee (Melipona scutellaris Latreille, 1811). Revista Brasileira de Biologia 61:63-67.

CARVALHO JÚNIOR, O. 1996. Dieta, padrões de atividade e de agrupamento do Mono-Carvoeiro (Brachyteles arachnoides) no Parque Estadual Carlos Botelho - SP. Dissertação de mestrado, Universidade Federal do Pará, Belém.

CASTRO, M.S. 2002. Bee fauna of some tropical and exotic fruits: potential pollinators and their conservation. In Pollination bees - The conservation link between agriculture and nature (P. Kevan \& V.L. ImperatrizFonseca, eds.). Ministry of Environment, Brasília, p.275-288.

CASTRO, S.L.R. 1998. Ecologia do tié-sangue, Ramphocelus bresilius (Aves: Emberezidae: Thraupinae), no Parque Estadual da Serra do Mar, Núcleo Picinguaba, Ubatuba, SP. Dissertação de mestrado, Universidade Estadual Paulista, Rio Claro.

CASTRO, E.R. \& GALETTI, M. 2004. Frugivoria e dispersão de sementes pelo lagarto Tupinambis merianae (Reptilia, Teiidae). Papéis Avulsos de Zoologia 44:91-97.

CHITOLINA, O.P. \& SANDER, M. 1981. Contribuição ao conhecimento da alimentação de Alouatta guariba clamitans Cabrera 1940 em habitat natural no Rio Grande do Sul (Cebidae, Alouattinae). Iheringia, Série Zoologia, 59:25-36.

CORDEIRO, N.J. \& HOWE, H.F. 2003. Forest fragmentation severs mutualism between seed dispersers and an endemic African tree. Proceedings of the National Academy of Science 100:14052-14056.

CORDERO R., G.A. \& NICOLAS B., R.A. 1987. Feeding habits of the Opossum (Didelphis marsupialis) in Northern Venezuela. Fieldiana, Zoology 39:125-131. 
CORTOPASSI-LAURINO, M. \& RAMALHO, M. 1988. Pollen harvest by africanized Apis mellifera and Trigona spinipes in São Paulo - Botanical and ecological views. Apidologie 19:1-24.

COSTA, C.P.A. 1998. Frugivoria e dispersão de sementes por quatis (Procyonidae, Nasua narica) no Parque das Mangabeiras, Belo Horizonte, MG. Dissertação de mestrado, Universidade Estadual de Campinas, Campinas.

CUNHA, A.S. 1994. Aspectos sócio-ecológicos de um grupo de bugios (Alouatta fusca clamitans) do Parque Estadual de Itapuã, RS. Dissertação de mestrado, Universidade Federal do Rio Grande do Sul, Porto Alegre.

DALPONTE, J.C. 1997. Diet of the hoary fox, Lycalopex vetulus, in Mato Grosso, Central Brazil. Mammalia 61:537-546.

DALPONTE, J.C. \& LIMA, E.S. 1999. Disponibilidade de frutos e a dieta de Lycalopex vetulus (Carnivora Canidae) em um cerrado de Mato Grosso, Brasil. Revista Brasileira de Botânica 22:325-332.

DEGENHART, J., ORTH, A.I., GUERRA, M.P., DUCROQUET, J.P. \& NODARI, R.O. 2001. Morfologia floral da goiabeira serrana (Feijoa sellowiana) e suas implicações na polinização. Revista Brasileira de Fruticultura 23:718-721.

DIETZ, J.M. 1984. Ecology and social organization of the Maned Wolf (Chrysocyon brachyurus). Smithsonian Contributions to Zoology 392:1-51.

DINERSTEIN, E. 1986. Reproductive ecology of fruit bats and the seasonality of fruit production in a Costa Rican cloud forest. Biotropica 18:307-318.

DUCROQUET, J.P.H.J. 1993. A pesquisa em goiabeira serrana (Feijoa sellowiana Berg) em Santa Catarina. In Anais do Simpósio Nacional de Recursos Genéticos de Fruteiras Nativas. Embrapa-CNPMF. Cruz das Almas, p.51-55.

DUCROQUET, J.P.H.J. \& HICKEL, E.R. 1997. Birds as pollinators of Feijoa (Acca sellowiana Berg). Acta Horticulturae 452:37-40.

ENDRESS, P.K. 1994. Diversity and evolutionary biology of tropical flowers. Cambridge University Press, Cambridge.

FACURE, K.G. \& GIARETTA, A.A. 1996. Food habits of carnivores in a Coastal Atlantic forest of southeastern Brazil. Mammalia 60:499-502.

FACURE, K.G. \& MONTEIRO FILHO, E.L.A. 1996. Feeding habits of the crab-eating fox, Cerdocyon thous (Carnivora, Canidae) in a suburban area of southeastern Brazil. Mammalia 60:147-149.

FALCÃO, M.A., CHAVEZ-FLORES, W.B., FERREIRA, S.A.N., CLEMENT, C.R., BARROS, M.J.B., BRITO, J.M.C. \& SANTOS, T.C.T. 1988. Aspectos fenológicos e ecológicos do “araçá-boi” (Eugenia stipitata McVaugh) na Amazônia Central. I. Plantas juvenis. Acta Amazonica 18:27-38.
FALCÃO, M.A., FERREIRA, S.A.N., CHAVEZ-FLORES, W.B. \& CLEMENT, C.R. 1989. Aspectos fenológicos e ecológicos do camu-camu (Myrciaria dubia (H.B.K.) McVaugh) na terra firme da Amazônia Central. In Anais do $10^{\circ}$ Congresso Brasileiro de Fruticultura (Sociedade Brasileira de Fruticultura, ed.). Fortaleza, p.59-64.

FALCÃO, M.A., FERREIRA, S.A.N., CLEMENT, C.R., SANTOS, T.C.T. \& SOUZA, R.M. 1992. Crescimento e fenologia de araçá-pera (Psidium acutangulum DC). Acta Amazonica 22:285-293.

FALCÃO, M.A., GALVÃO, R.M.S., CLEMENT, C.R., FERREIRA, S.A.N. \& SAMPAIO, S.G. 2000. Fenologia e produtividade do araçá-boi (Eugenia stipitata, Myrtaceae) na Amazônia Central. Acta Amazonica 30:9-21.

FIALHO, M.S. \& SETZ, E.Z.F. 2000. Brown howler seasonal frugivory in extreme southern Brazil. In III International Symposium - Workshop on Frugivores and Seed Dispersal Biodiversity and Conservational Perspectives. Proceedings of III International Symposium - Workshop on Frugivores and Seed Dispersal Biodiversity and Conservational Perspectives. São Pedro, v.1., p.171-171.

FIDALGO, A.O. 2002. Interação entre abelhas e plantas da família Myrtaceae numa floresta de planície litorânea em Ubatuba - SP, Brasil. Tese de doutorado, Universidade de São Paulo, São Paulo.

FLEMING, T.H. 1988. The short-tailed fruit bat: a study in plant-animal interactions. University of Chicago Press, Chicago.

FONSECA, G.A.B. 1985. The vanishing Brazilian Atlantic Forest. Biological Conservation 34:17-34.

FORGET, P.M. \& MILLERON, T. 1991. Evidence for secondary seed dispersal by rodents in Panama. Oecologia 87:596-599.

GALETTI, M. \& PIZO, M.A. 1996. Fruit eating by birds in a forest fragment in southeastern Brazil. Ararajuba 4:71-79.

GALETTI, M., MARTUSCELLI, P., OLMOS, F. \& ALEIXO, A. 1997. Ecology and conservation of Jacutinga Pipile jacutinga in the Atlantic forest of Brazil. Biological Conservation 82:31-39.

GASPAR, D.A. 1997. Ecologia e comportamento do bugio ruivo, Alouatta fusca (Geoffroy, 1812, Primates: Cebidae), em um fragmento de mata de Campinas, SP. Dissertação de mestrado, Universidade Estadual Paulista, Rio Claro.

GOTTSBERGER, G. \& SILBERBAUER-GOTTSBERGER, I. 1983. Dispersal and distribution of plants in the cerrado vegetation of Brazil. Sonderbänd des Naturwissenschaftlichen Vereins in Hamburg 7:315-352.

GRESSLER, E. 2005. Floração e frutificação de Myrtaceae de floresta atlântica: limitações ecológicas e filogenéticas. Dissertação de mestrado, Universidade Estadual Paulista, Rio Claro.

GRIFO, F.T. 1992. Arevision of Myrcianthes Berg (Myrtaceae). PhD. Dissertation, University of Cornell, Ithaca. 
GUIBU, L.S., RAMALHO, M., KLEINERT-GIOVANNINI, A. \& IMPERATRIZ-FONSECA, V.L. 1988. Exploração de recursos florais por colônias de Melipona quadrifasciata (Apidae, Meliponinae). Revista Brasileira de Biologia 48:299-305.

GUILHERME, F.A.G., MORELLATO, L.P.C. \& ASSIS, M.A. 2004. Horizontal and vertical tree community structure in a lowland Atlantic rain forest, Southeastern Brazil. Revista Brasileira de Botânica 27:725-737.

GUIX, J.C. \& RUIZ, X. 1998. Intensive flolivory by Thraupis sayaca (Emberezidae: Thraupinae) in southeastern Brazil. Ararajuba 6:138-140.

HASUI, E. \& HÖFLING, E. 1998. Preferência alimentar das aves frugívoras de um fragmento de floresta estacional semidecídua secundária, São Paulo, Brasil. Iheringia, Série Zoologia 84:43-64.

HICKEL, E.R. \& DUCROQUET, J.P.J. 2000. Polinização entomófila da goiabeira serrana, Feijoa sellowiana (Berg), em Santa Catarina. Revista Brasileira de Fruticultura 22:96-101.

HOPPER, S.D. 1980. Pollination of the rain-forest tree Syzygium tierneyanum (Myrtaceae) at Kuranda, Northern Queensland. Australian Journal of Botany 28:223-237.

IMPERATRIZ-FONSECA, V.L., KLEINERT-GIOVANNINI, A. \& RAMALHO, M. \& 1989. Pollen harvest by eusocial bees in a non-natural community in Brazil. Journal of Tropical Ecology 5:239-242.

IMPERATRIZ-FONSECA, V.L., RAMALHO, M. \& KLEINERT-GIOVANNINI, A. 1993. Abelhas sociais e flores: análise polínica como método de estudo. In Flores e abelhas em São Paulo (J.R. Pirani \& M. CortopassiLaurino, eds.). Edusp/ Fapesp, São Paulo, p.17-30.

IZAR, P. 1999. Aspectos de ecologia e comportamento de um grupo de macacos-prego (Cebus apella) em área de Mata Atlântica, São Paulo. Tese de doutorado, Universidade de São Paulo, São Paulo.

KAWASAKI, M.L. \& LANDRUM, L.R. 1997. A rare and potentially economic fruit of Brazil - cambuci, Campomanesia phaea (Myrtaceae). Economic Botany 51:403-407.

KINDEL, A. 1996. Interações entre plantas ornitocóricas e aves frugívoras na estação ecológica de Aracuri, Muitos Capões, RS. Dissertação de Mestrado, Universidade Federal do Rio Grande do Sul, Porto Alegre.

KEELER-WOLF, T. 1988. Fruit and consumer differences in three species of trees shared by Trinidad and Tobago. Biotropica 20:38-48.

KNOLL, F.R.N. \& IMPERATRIZ-FONSECA, V.L. 1987. Abundance and resources visited by individuals of Tetragonisca angustula. In Chemistry and Biology of Social Insects (J. Eder \& H. Rembold, orgs.). Verlag J. Peperny, Müchen, p.702-703.
KNOLL, F.R.N., REGO, L.R. \& IMPERATRIZ-FONSECA, V.L. 1993. As abelhas em áreas urbanas - um estudo no Campus da Universidade de São Paulo. In Flores e abelhas em São Paulo (J.R. Pirani \& M. CortopassiLaurino, eds.). Edusp/ Fapesp, São Paulo, p.31-42.

KUBITZKI, K. \& ZIBURSKI, A. 1994. Seed dispersal in flood plain forests of Amazonia. Biotropica 26:30-43.

KUHLMANN, M. 1975. Adendo alimentar dos bugios. Silvicultura de São Paulo 9:57-62.

KUHLMANN, M. \& KUHN, E. 1947. A flora do Distrito de Ibiti. Instituto de Botânica, São Paulo.

LANDRUM, L.R. 1986. Campomanesia, Pimenta, Blepharocalyx, Legrandia, Acca, Myrrhinium, and Luma (Myrtaceae). Flora Neotropica Monographs 45. New York Botanical Garden, New York.

LANDRUM, L.R. \& KAWASAKI, M.L. 1997. The genera of Myrtaceae in Brazil - an illustrated synoptic treatment and identification keys. Brittonia 49:508-536.

LANDRUM, L.R. \& SHARP, W.P. 1989. Seed coat characters of some American Myrtinae (Myrtaceae): Psidium and related genera. Systematic Botany 14:370-376.

LOPES, M.A. \& FERRARI, S.F. 1994. Foraging behavior of a tamarin group (Saguinus fuscicollis weddelli) and interactions with marmosets. International Journal of Primatology 15:373-387.

LORENZI, H. 1998. Árvores brasileiras. $2^{\text {a }}$ ed. Instituto Plantarum, Nova Odessa, v.1.

MACEDO, M. 1977. Dispersão de plantas lenhosas de uma campina Amazônica. Acta Amazonica 7:1-69.

MALERBO, D.T.S., TOLEDO, V.A.A. \& COUTO, R.H.N. 1991. Polinização entomófila em jabuticabeira (Myrciaria cauliflora Berg.). Ciência Zootécnica - Jaboticabal 6:3-5.

MANHÃES, M.A. 2003. Dieta de Traupíneos (Passeriformes, Emberizidae) no Parque Estadual do Ibitipoca, Minas Gerais, Brasil. Iheringia, Série Zoologia 93:59-73.

MARCHIORI, J.N.C. \& SOBRAL, M. 1997. Dendrologia das angiospermas - Myrtales. Editora da UFSM, Santa Maria.

MARQUEZ-SOUZA, A.C. 1996. Fontes de pólen exploradas por Melipona compressipes manaosensis (Apidae: Meliponinae), abelha da Amazônia Central. Acta Amazonica 26:77-86.

MARSHALL, A.G. 1985. Old world phytophagous bats (Megachiroptera) and their food plants: a survey. Zoological Journal of the Linnean Society 83:351-369.

MARTINS, C.S. 1997. Uso do habitat pelo bugio, Alouatta fusca clamitans, em um fragmento florestal em Lençois Paulista-SP. Dissertação de mestrado, Universidade Estadual de Campinas, Campinas.

MARTUSCELLI, P. \& OLMOS, F. 1997. Cracid conservation in São Paulo State, Southeastern Brazil. In The Cracidae: their biology and conservation (S.D. Strahl, S. Beaujon, D.M. Brooks, A.J. Begazo, G. Sedaghatkish \& F. Olmos, eds.). Hancock House Publishers, Blaine, p.482-491. 
MATTOS, J.R. 1986. A goiabeira serrana. Instituto de Pesquisas de Recursos Naturais Renováveis, Porto Alegre.

MAUÉS, M.M. \& COUTURIER, G. 2002. Biologia floral e fenologia reprodutiva do camu-camu (Myrciaria dubia (H.B.K.) McVaugh, Myrtaceae) no Estado do Pará, Brasil. Revista Brasileira de Botânica 25:441-448.

MENDONÇA-LIMA, A., FONTANA, C.S. \& MÄHLER JÚNIOR, J.K.F. 2001. Itens alimentares consumidos por aves no nordeste do Rio Grande do Sul. Tangara 1:115-124.

MERCOLLI, C. \& YANOSKY, A.A. 1990. Repértoire de comportaments du teju (Tupinambis teguixin), Sáuria: Teiidae. Revue Africaine Aquaridologie 16:123-130.

MIKICH, S.B. 2000. Frugivoria e dispersão de sementes em uma pequena reserva isolada no estado do Paraná, Brasil. Tese de doutorado, Universidade Estadual de Campinas, Campinas.

MIRANDA, J.M.D. \& PASSOS, F.C. 2004. Hábito alimentar de Alouatta guariba (Humboldt) (Primates, Atelidae) em floresta de Araucária, Paraná, Brasil. Revista Brasileira de Zoologia 21:821-826.

MORAES, P.L.R. 1992a. Dispersão de sementes pelo monocarvoeiro (Brachyteles arachnoides E. Geoffroy, 1806) no Parque Estadual de Carlos Botelho. Revista do Instituto Florestal 4:1193-1208.

MORAES, P.L.R. 1992b. Espécies utilizadas na alimentação do Mono-Carvoeiro (Brachyteles arachnoides E. Geoffroy, 1806) no Parque Estadual de Carlos Botelho. In II Congresso Nacional sobre Essências Nativas. Revista do Instituto Florestal 4:1206-1208.

MORELLATO, L.P.C. \& HADDAD, C.F.B. 2000. Introduction: The Brazilian Atlantic forest. Biotropica 32:786-792.

MOTTA JÚNIOR, J.C., LOMBARDI, J.A. \& TALAMONI, S.A. 1994. Notes on crab-eating fox (Dusicyon thous) seed dispersal and food habits in southeastern Brazil. Mammalia 58:156-159.

MOTTAJÚNIOR, J.C. \& MARTINS, K. 2002. The frugivorous diet of the Maned Wolf, Chrysocyon brachyurus, in Brazil - ecology and conservation. In Frugivory and seed dispersal - evolution, ecology and conservation (D.J. Levey, W.R. Silva \& M. Galetti, eds.). CAB International, Wallingford, p.291-303.

NIC LUGHADHA, E.N. \& PROENÇA, C. 1996. A survey of the reproductive biology of the Myrtoideae (Myrtaceae). Annals of the Missouri Botanical Garden 83:480-503.

NUNES, A. 1998. Diet and feeding ecology of Ateles belzebuth belzebuth at Maracá Ecological Station, Roraima, Brazil. Folia Primatologica 49:11-32

OLIVEIRA FILHO, A.T. \& FONTES, M.A. 2000. Patterns of floristic differentiation among Atlantic forests in southeastern Brazil, and the influence of climate. Biotropica 32:793-810.
OLIVEIRA, P.E. \& GIBBS, P.E. 2000. Reproductive biology of woody plants in a cerrado community of Central Brazil. Flora 195:311-329.

OLIVEIRA, P.E. \& GIBBS, P.E. 2002. Pollination and reproductive biology in cerrado plant communities. In The cerrados of Brazil - ecology and natural history of a neotropical savanna (P.S. Oliveira \& R.J. Marquis, eds.), Columbia University Press, New York, p.329-347.

OLIVEIRA, P.E.A.M. \& PAULA, F.R. 2001. Fenologia e biologia reprodutiva de plantas de matas de galeria. In Cerrado - caracterização e recuperação de matas de galeria (Embrapa-CPAC, ed.). Brasília, p.303-332.

PACK, K.S., HENRY, O. \& SABATIER, D. 1996. The insectivorous-frugivorous diet of the Golden-handed Tamarin (Saguinus midas midas) in French Guiana. Folia Primatologica 70:1-7.

PASSOS, F.C. 1992. Hábito alimentar do mico-leão-preto Leontopithecus chrysopygus (Mikan, 1823) (Callithricidae, Primates) na estação ecológica de Caetetus, município de Gália, SP. Dissertação de mestrado, Universidade Estadual de Campinas, Campinas.

PASSOS, F.C. 1997. Padrão de atividades, dieta e uso do espaço em um grupo de mico-leão-preto (Leontopithecus chrysopygus) na estação ecológica de Caetetus, SP. Tese de doutorado, Universidade Estadual de Campinas, Campinas.

PASSOS, L.C. \& OLIVEIRA, P.S. 2003. Interactions between ants, fruits and seeds in a restinga forest in southeastern Brazil. Journal of Tropical Ecology 19:261-270.

PEIXOTO, A.L. \& GENTRY, A.H. 1990. Diversidade e composição florística da mata de tabuleiro na Reserva Florestal de Linhares (Espírito Santo, Brasil). Revista Brasileira de Botânica 13:19-25.

PEREIRA, M.V.L., PEIXOTO, A.L. \& DI MAIO, F.R. 1995. Plantas utilizadas como recurso alimentar pela fauna silvestre na represa de Ribeirão das Lajes, Rio de Janeiro, Brasil. Revista da Universidade Rural, Série Ciências da Vida 17:25-40.

PETERS, C.M. \& VASQUEZ, A. 1986-1987. Estudios ecológicos de camu-camu (Myrciaria dubia). I. Producción de frutos en poblaciones naturales. Acta Amazonica 16/17:161-174.

PETRONI, L.M. 1993. Aspectos da ecologia e comportamento do Mono Carvoeiro Brachyteles arachnoides (E. Geoffroy, 1806) (Cebidae, Primates), na Fazenda Intervales, Serra de Paranapiacaba, São Paulo. Dissertação de mestrado, Pontifícia Universidade Católica do Rio Grande do Sul, Porto Alegre.

PETRONI, L.M. 2000. Caracterização da área de uso e dieta do mono carvoeiro (Brachyteles arachnoides, Cebidae - Primates), na Mata Atlântica, Serra de Paranapiacaba, SP. Tese de doutorado, Universidade de São Paulo, São Paulo. 
PIRANI, J.R. \& CORTOPASSI-LAURINO, M. 1993. Flores e abelhas em São Paulo. Edusp/Fapesp, São Paulo.

PIRES, A.F. 1997. Dispersão de sementes na várzea do médio Solimões, Estado do Amazonas, Brasil. Dissertação de mestrado, Universidade Federal do Pará/ Museu Paraense Emílio Goeldi, Belém.

PIZO, M.A. 2002. The seed dispersers and fruit syndromes of Myrtaceae in Brazilian Atlantic forest. In Frugivores and seed dispersers - biodiversity and conservation perspectives. (D.J. Levey, W.R. Silva \& M. Galetti, eds.), CABI Publishing, Wallingford, p.129-143.

PIZO, M.A. 2003. Padrão de deposição de sementes e sobrevivência de sementes e plântulas de duas espécies de Myrtaceae na Mata Atlântica. Revista Brasileira de Botânica 26:371-377.

PIZO, M.A. \& OLIVEIRA, P.S. 2000. The use of fruits and seeds by ants in the Atlantic forest of Southeast Brazil. Biotropica 32:842-850.

POPENOE, F.W. 1912. Feijoa sellowiana, its history, culture and varieties. Pomona College Journal of Economic Botany 2:217-242.

PROENÇA, C.E.B. 1992. Buzz pollination - older and more widespread than we think? Journal of Tropical Ecology 8:115-120.

PROENÇA, C.E.B. \& GIBBS, P.E. 1994. Reproductive biology of eight sympatric Myrtaceae from Central Brasil. New Phytologist 126:343-354.

RAMALHO, M. 1990. Foraging by stingless bees of the genus Scaptotrigona (Apidae, Meliponinae). Journal of Apicultural Research 29:61-67.

RAMALHO, M. 2004. Stingless bees and mass flowering trees in the canopy of Atlantic Forest - a tight relationship. Acta Botanica Brasilica 18:37-47.

RAMALHO, M., KLEINERT-GIOVANNINI, A. \& IMPERATRIZ-FONSECA, V.L. 1989. Utilization of floral resources by species of Melipona (Apidae, Meliponinae): floral preferences. Apidologie 20:185-195.

RAMALHO, M., KLEINERT-GIOVANNINI, A. \& IMPERATRIZ-FONSECA, V.L. 1990. Important bee plants for stingless bees (Melipona and Trigonini) and africanized honeybees (Apis mellifera) in neotropical habitats: a review. Apidologie 21:469-488.

RANTA, P., BLOM, T., NIEMELÄ, J., JOENSUU, E. \& SIITINEN, M. 1998. The fragmented Atlantic rain forest of Brazil: size, shape and distribution of forest fragments. Biodiversity Conservation 7:385-403.

REDFORD, K.H. 1992. The empty forest. Bioscience 42:412-422.

RILEY, C.M. \& SMITH, K.G. 1992. Sexual dimorphism and foraging behavior of Emerald Toucanets Aulacorhynchus prasinus in Costa Rica. Ornis Scandinavica 23:459-466.

ROITMAN, G.G., MONTALDO, N.H. \& MEDAN, D. 1997. Pollination biology of Myrrhinium atropurpureum (Myrtaceae): sweet, fleshy petals attract frugivorous birds. Biotropica 29:162-168.
ROTH, I. 1987. Stratification of a tropical forest as seen in dispersal types. Kluwer Academic Publisher, Dordrecht.

ROUBIK, D.W. 1978. Competitive interactions between neotropical pollinators and africanized honeybees. Science 201:1030-1032.

ROUBIK, D.W. 1979. Africanized honeybees, stingless bees and the structure of tropical plant pollinator comunities. In Proceedings of the IV International Symposium on Pollination. (D. Caron, ed.). Maryland Agricultural Experimental Station, Maryland, p.403-417.

ROUBIK, D.W. 1989. Ecology and natural history of tropical bees. Cambridge University Press, Cambridge.

ROUBIK, D.W., MORENO, J.E., VERGARA, C. \& WITTMANN, D. 1986. Sporadic food competition with the African honeybee: projected impact on neotropical social bees. Journal of Tropical Ecology 2:97-111.

SAZIMA, M. \& SAZIMA, I. 1999. The perching bird Coereba flaveola as a co-pollinator of bromeliad flowers in Southeastern Brazil. Canadian Journal of Zoology 77:47-51.

SAZIMA, I., BUZATO, S. \& SAZIMA, M. 1993. The bizarre inflorescence of Norantea brasiliensis (Marcgraviaceae) - visits of hovering and perching birds. Botanica Acta 106:507-513.

SCHAFFER, W.M., ZEH, D.W., BUCHMANN, S.L., KLEINHAUS, I., SCHAFFER, M.V. \&ANTRIN, J. 1983. Competition for nectar between introduced honeybees and native North American bees and ants. Ecology 64:564-577.

SILBERBAUER-GOTTSBERGER, I. \& GOTTSBERGER, G. 1988. A polinização de plantas do cerrado. Revista Brasileira de Biologia 48:651-663.

SILVA, J.M.C. \& TABARELLI, M. 2000. Tree species impoverishment and the future flora of the Atlantic forest of Northeast Brazil. Nature 404:72-74.

SNOW, B.K. \& SNOW, D.W. 1971. The feeding ecology of tanagers and honeycreepers in Trinidad. Auk 88:291-322.

SNOW, D.W. 1962. A field study of the black and white Manakin, Manacus manacus, in Trinidad. Zoologica 47:65-104.

SOINI, P. 1987. Ecology of the saddle-back Tamarin Saguinus fuscicollis illigeri on the Rio Pacaya, Northeastern Peru. Folia Primatologica 49:11-32.

SOUZA, L.L., FERRARI, S.F. \& PINA, A.L.C.B. 1997. Feeding behaviour and predation of a bat by Saimiri sciurus in a semi-natural Amazonian environment. Folia Primatologica 68:194-198.

SOUZA, M.A.D. 1997. Biologia reprodutiva de onze espécies de Myrtaceae em floresta de terra firme na Amazônia Central. Dissertação de mestrado, Instituto Nacional de Pesquisas da Amazônia/Universidade Federal do Amazonas, Manaus. 
SOUZA, V.C., CORTOPASSI-LAURINO, M., SIMÃOBIANCHINI, R., PIRANI, J.R., AZOUBEL, M.L., GUIBU, L.S. \& GIANNINI, T.C. 1993. Plantas apícolas de São Paulo e arredores. In Flores e abelhas em São Paulo. (J.R. Pirani \& M. Cortopassi-Laurino, eds.). Edusp/ Fapesp, São Paulo, p.43-179.

STEINMETZ, S. 2000. Ecologia e comportamento do bugio (Alouatta fusca clamitans, Atelidae - Primates) no Parque Estadual Intervales - SP. Dissertação de mestrado, Universidade de São Paulo, São Paulo.

STEWART, P. 1986. Birds, not bees for feijoas. Horticulture News 8:20.

STEWART, A.M. 1987. Reproductive biology and pollination ecology of Feijoa sellowiana. PhD Thesis, University of Auckland, Auckland.

STRIER, K.B. 1991. Diet of one group of Wooly Spider Monkeys, or Muriquis (Brachyteles arachnoides). American Journal of Primatology 23:113-126.

SUGAHARA, V.Y. \& TAKAKI, M. 2004. Effect of light and temperature on seed germination in guava (Psidium guajava L., Myrtaceae). Seed Science and Technology 32:759-764.

TABARELLI, M. \& MANTOVANI, W. 1999. A riqueza de espécies arbóreas na floresta atlântica de encosta no estado de São Paulo (Brasil). Revista Brasileira de Botânica 22:217-223.

TERBORGH, J. 1983. Five new world primates: a study in comparative ecology. Princeton University Press, Princeton.

THÉRY, M. \& LARPIN, D. 1993. Seed dispersal and vegetation dynamics at a Cock-of-the-rock's lek in the tropical forest of French Guiana. Journal of Tropical Ecology 9:109-116.
THÉRY, M., ÉRARD, C. \& SABATIER, D. 1991. Les fruits dans le regime alimentaire de Penelope marail (Aves, Cracidae) en forêt Guyanaise: frugivore stricte et selective? Revue D'Ecologie 47:383-401.

TORENZAN-SILINGARDI, H.M. \& DEL-CLARO, K. 1998. Behavior of visitors and reproductive biology of Campomanesia pubescens (Myrtaceae) in cerrado vegetation. Ciência e Cultura 50:281-284.

TORENZAN-SILINGARDI, H.M. \& OLIVEIRA, P.E.A.M. 2004. Phenology and reproductive ecology of Myrcia rostrata and M. tomentosa (Myrtaceae) in Central Brazil. Phyton (Horn) 44:23-43.

VAN DER PIJL, L. 1982. Principles of seed dispersal in higher plants. Springer-Velag, Berlin.

VAN ROOSMALEM, M.G.M. 1985a. Fruits of the Guianan Flora. Drukkerij Veenman B.V., Wageningen.

VAN ROOSMALEM, M.G.M. 1985b. Habitat preferences, diet, feeding strategy and social organization of the black spider monkey (Ateles paniscus paniscus Linnaeus 1758) in Surinam. Acta Amazonica 15:1-238.

VAN WYK, A.E. \& LOWREY, T.K. 1988. Studies on the reproductive biology of Eugenia L. (Myrtaceae) in Southern Africa. Monographs in Systematic Botany from the Missouri Botanical Garden 25:279-293.

VOSS, W.A. \& SANDER, M. 1980. Frutos e árvores nativas na alimentação das aves. Trigo e Soja 51:26-30.

WHEELWRIGHT, N.T. 1985. Fruit size, gape width, and the diets of fruit-eating birds. Ecology 66:808-818.

WILLIS, E.O. 1979. The composition of avian communities in reminiscent woodlots in Southern Brazil. Papéis Avulsos de Zoologia 33:1-25.

WILSON, P.G., O'BRIEN, M.M., HESLEWOOD, M.M. \& QUINN, C.J. 2005. Relationships within Myrtaceae sensu lato based on a matK phylogeny. Plant Systematics and Evolution 251:3-19. 